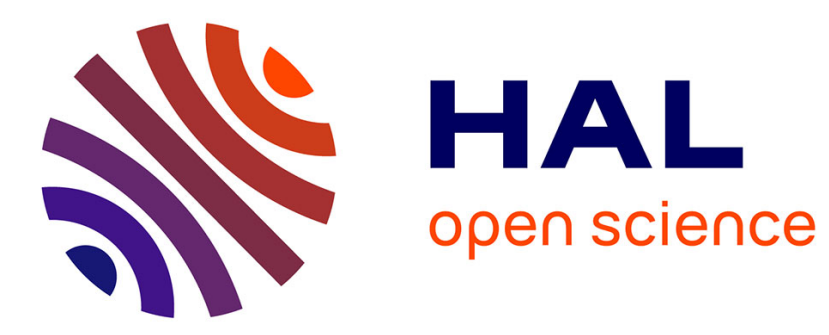

\title{
On flow-acoustic resonant interactions in transitional airfoils
}

Vladimir V. Golubev, Lap Nguyen, Reda R. Mankbadi, Michel Roger, Miguel

R. Visbal

\section{- To cite this version:}

Vladimir V. Golubev, Lap Nguyen, Reda R. Mankbadi, Michel Roger, Miguel R. Visbal. On flowacoustic resonant interactions in transitional airfoils. International Journal of Aeroacoustics, 2014, 13 (1-2), pp.1-38. 10.1260/1475-472X.13.1-2.1 . hal-01136616

\section{HAL Id: hal-01136616 https://hal.science/hal-01136616}

Submitted on 11 Apr 2016

HAL is a multi-disciplinary open access archive for the deposit and dissemination of scientific research documents, whether they are published or not. The documents may come from teaching and research institutions in France or abroad, or from public or private research centers.
L'archive ouverte pluridisciplinaire HAL, est destinée au dépôt et à la diffusion de documents scientifiques de niveau recherche, publiés ou non, émanant des établissements d'enseignement et de recherche français ou étrangers, des laboratoires publics ou privés. 


\title{
On flow-acoustic resonant interactions in transitional airfoils
}

\author{
Vladimir V. Golubev'1, Lap Nguyen', Reda R. Mankbadi', Michel \\ Roger ${ }^{2}$ and Miguel R. Visbal ${ }^{3}$ \\ IFlorida Center for Advanced Aero Propulsion, Computational Aero Propulsion Group \\ Embry-Riddle Aeronautical University, Daytona Beach, FL 32174, USA \\ (Vladimir.Golubev@erau.edu,nguye276@erau.edu,mankbadr@erau.edu) \\ 2Laboratoire de Mecanique des Fluides et Acoustique \\ Ecole Centrale de Lyon, Ecully 69134, France \\ (MichelRoger@ec-lyon.fr) \\ ${ }^{3}$ Air Force Research Laboratory, WPAFB, OH 80906, USA \\ (miguel.visba@@wpafb.af.mil)
}

Received: Feb. 4, 2013; Revised: March 3, 2014; Accepted: March 5, 2014

\begin{abstract}
We discuss our recent experimental and preliminary numerical efforts examining resonant feedback mechanism of flow-acoustic interactions in airfoil transitional boundary layers. Experimentally recorded unsteady responses of loaded, transitional NACA0012 airfoil with tripped suction or pressure sides confirm the presence of shifted ladder-type tonal structures with dual velocity dependence in the acoustic signal previously reported in the literature. The installation of upstream grid generating low-intensity turbulence appears to eliminate the feedback mechanism leaving just a single velocity dependence for the dominant spectral components. Complementary numerical efforts employ a high-order Navier-Stokes solver implementing low-pass filtering of poorly resolved high-frequency solution content to retain numerical accuracy and stability over the range of transitional flow regimes. Within the scope of 2D analysis, the conducted numerical experiments particularly investigate the behavior of the boundary-layer statistical moments during the transitional flow regimes characterized by the presence of the acoustic feedback, and address sensitivity of the latter to the flow Reynolds number.
\end{abstract}

\section{INTRODUCTION}

For an airfoil entering a region of non-uniform unsteady flow, its unsteady aerodynamic response and noise radiation pattern generally exhibit the interference of both leadingedge (LE) and trailing-edge (TE) sources. The former are dominant in high-speed turbomachinery applications and particularly in the area of unsteady rotor-stator interactions. They have been extensively studied, e.g., in the context of inviscid gustairfoil interaction problem. It is suggested that the impact of impinging upstream 
disturbances may interfere with or even trigger the trailing edge noise sources, e.g., through the enhanced boundary layer dynamics. High-accuracy viscous analyses of Golubev et al ${ }^{1,2}$ initiated a numerical study of the interference process by comparing of the unsteady aerodynamic and acoustic responses of a symmetric Joukowski airfoil with and without impinging vortical gust harmonic. Results obtained for selected chord-based flow Reynolds numbers in the range from $\operatorname{Re}_{c}=10^{4}$ to $\operatorname{Re}_{c}=2 \times 10^{6}$ revealed a strong gust phase-locking mechanism which produced a major impact on the boundary-layer vorticity dynamics and the resulting RMS surface pressure levels by exciting a timeperiodic generation of the vortical structures with the frequency of the gust on both sides of the airfoil.

On the other hand, with clean upstream flow conditions, the dominant mechanism of the trailing edge noise radiation associated, e.g., with acoustics of high-lift devices as well as low-speed airfoils, rotors, fans and wind turbine blades, is commonly related to the viscous dynamics of the convected boundary layer structures scattering into acoustics at the airfoil trailing edge. In this case, the airfoil trailing-edge unsteady response appears particularly pronounced in the transitional flow regimes. Such behavior, in fact, could be linked to the appearance of flow-acoustic resonant interaction phenomena associated with the acoustic feedback loop between the trailingedge acoustic waves and the excited boundary-layer instability waves amplifying and scattering as sound at the airfoil trailing edge.

The first comprehensive experimental study by Paterson et $\mathrm{al}^{3}$ focused on tonal noise emitted by symmetric NACA airfoils in a Reynolds number range corresponding to full-scale helicopter rotors. Their key finding reproduced in Fig. 1 shows an unusual ladder-type structure (staging) of tones wherein the peak frequencies are scaled $\sim U^{1.5}$ ( $U$ is the free-stream velocity) with sudden jumps appearing between the rungs of the ladder scaled $\sim U^{0.8}$. Tam ${ }^{4}$ proposed a feedback-loop mechanism to explain the tonal staging, with his original scenario of localized wake source corrected and further elaborated by Arbey \& Bataille $^{5}$ based on a series of experiments with NACA0012 airfoils. Their recorded acoustic spectra generally confirmed the tonal ladder structure of Paterson et $\mathrm{al}^{3}$ to produce, for a given velocity in the range of $U=20 \ldots 40 \mathrm{~m} / \mathrm{s}$ $\left(R e_{c}=2 \ldots 6 \times 10^{5}\right)$, a narrowband set of equidistant frequencies superimposed on a broadband hump (Fig. 2). In the interpretation first suggested by Longhouse ${ }^{6}$, such contribution was attributed to Tollmien-Schlichting (T-S) instability waves developing in the boundary layer and scattering as sound at the airfoil trailing edge. The acoustic feedback loop was presumed to be generated between selected trailing-edge frequency tones and the most amplified T-S wave based on the condition that both sound and convected waves are in phase at the point of the instability appearance. The existence of such mechanism was later examined theoretically by Atassi ${ }^{7}$ using the triple-deck theory approach.

Based on the adopted view of the feedback loop shown in Fig. 3, much of the later efforts focused on examining possible conditions and scenarios for evolution of instability waves and particularly the role of laminar separation regions in the wave amplification process. Despite a limited scope of such analyses in the context of the 


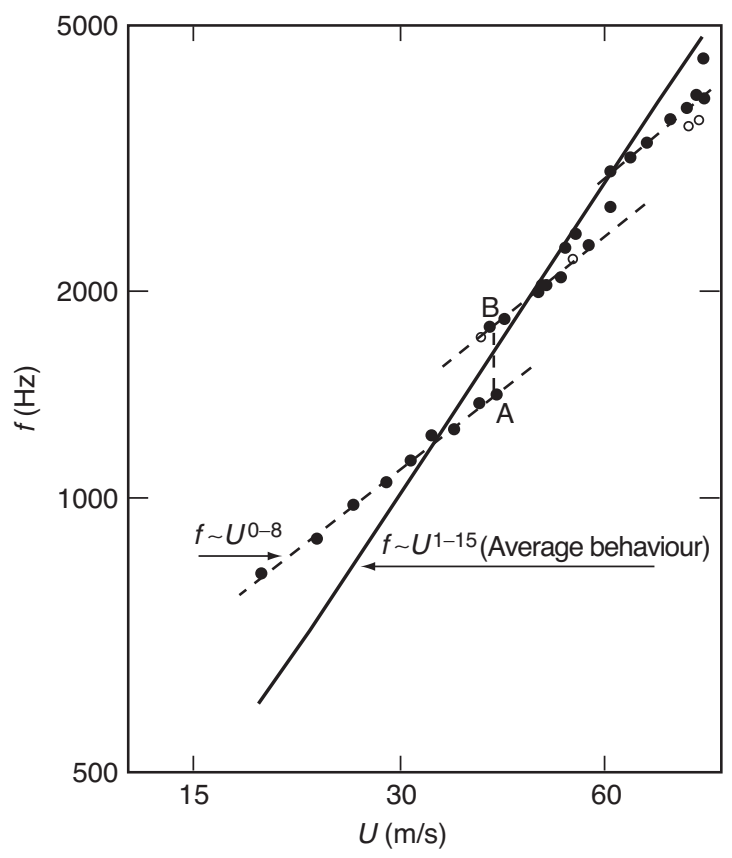

Figure 1: Airfoil ladder-type frequency structure, from Paterson et $\mathrm{al}^{3}$.

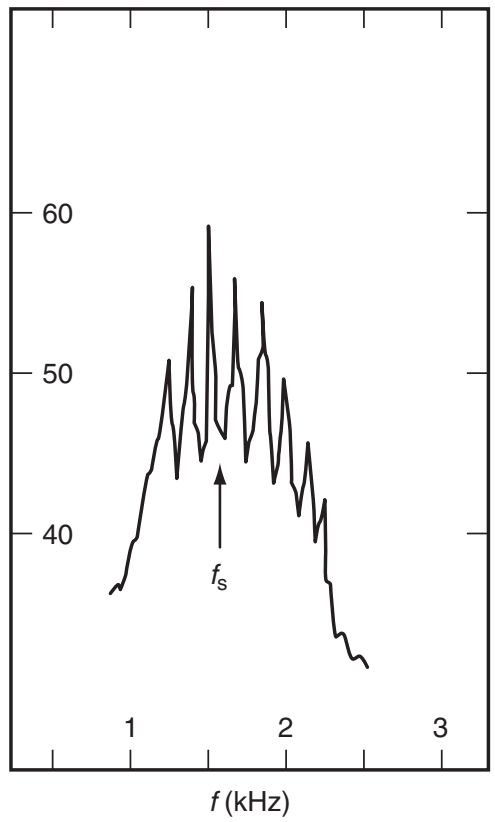

Figure 2: $\quad$ Airfoil frequency spectrum, from Arbey \& Bataille 5 . 


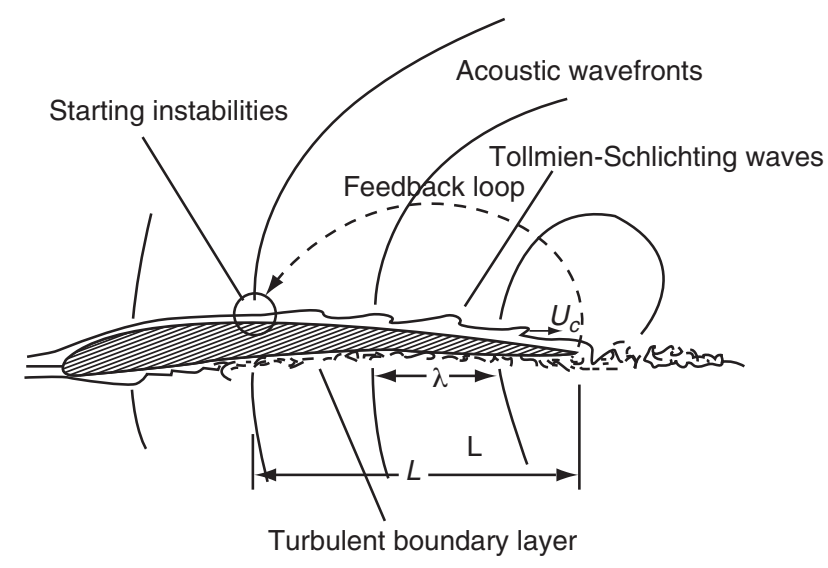

Figure 3: Feedback loop in airfoil flow-acoustic interaction, from Longhouse ${ }^{6}$.

overall problem, significant contradictions appear between different views on the basic physical mechanisms involved.

Lowson et $\mathrm{al}^{8}$ analyzed various experimental data for the NACA0012 airfoil to map regions of discrete tones in the Reynolds number/Angle of attack plane. They observed T-S waves at lower Reynolds numbers than the first tone appearance. In other words, the instability was not a sufficient condition for the occurrence of the acoustic radiation and/or the feedback process. Instead, such process was associated with the presence of a separation bubble at the airfoil pressure side, while the sound intensity was correlated with the length of the bubble. Nash et $\mathrm{al}^{9}$ continued with a series of experiments on a transitional NACA0012 airfoil. However, in contrast to Paterson et $\mathrm{al}^{3}$ and Arbey \& Bataille ${ }^{5}$, they failed to observe any ladder-type structure of acoustic tones (i.e., only single tonal frequencies for each flow case, aligned $\sim U^{0.8}$ ). They attributed such behavior to a cleaner experiment not contaminated by traverse acoustic duct modes in the wind tunnel (a point contested later on). Using LDA measurements, they observed cases with large regions of reversed flow without tones, but at the same time confirmed Lowson et $\mathrm{al}^{8}$ 's conjecture that a region of separated flow with inflectional flow profile near the trailing edge appears essential to produce tonegenerating amplified T-S instability. LDA results and smoke flow visualizations showed that such tonal cases were characterized by triple-peaked distributions of RMS velocity fluctuation profile in the boundary layer as well as regular vortex streets shed from the trailing edge.

Perhaps the focal point of most previous research on the subject was determining the frequency of the trailing-edge tones and its link to the suspected mechanism of T-S wave amplification. Here, a controversy between different works is apparent. Arbey \& Bataille ${ }^{5}$ employed neutral stability curves obtained by Obremsky et $\mathrm{al}^{10}$ to show that the Strouhal number (based on boundary-layer displacement thickness at trailing edge $\delta$ ) for maximum tonal radiation is independent of Reynolds number and given by $S t_{\delta}$ $\approx 0.048$. This, in fact, recovers $f_{s} \sim U^{1.5}$ dependence shown first by Paterson et al ${ }^{3}$ and 
consistent with vortex-shedding model of acoustic source. Nash et $\mathrm{al}^{9}$ developed a more accurate approach for separated flows to predict T-S wave amplifications based on solutions to Orr-Sommerfeld equations with input of Falkner-Scan's Hartree velocity profiles at each station along the airfoil. The transition from T-S waves to inviscid Rayleigh waves arising from inflectional profiles in separation region appeared critical in the amplification process. Hence, the effectively proposed scenario was that the produced tonal frequency corresponded to that of the (originally T-S) instability wave with maximum total amplification achieved before entering the separated region where inflectional instability with much higher growth rate took over. Although the exact connection between such mechanism produced by instability scattering and $f \sim U^{0.8}$ dependence was not discussed, the companion paper by McAlpine et $\mathrm{al}^{11}$ also suggested (based on the same experiments) that the observed vortex shedding at the pressure side owing to the separation bubble acted similar to the vortex shedding behind a cylinder. Such argument, however, would rather support $f \sim U^{1.5}$ dependence (a contradiction also noticed by McAlpine et $\mathrm{al}^{11}$ ). In fact, the vortical structures shed at the trailing edge could be linked to a region of absolute instability close to the body noted by Koch ${ }^{12}$ and later by Jones et $\mathrm{al}^{13}$ in reference to the view on the vortex shedding as a self-excited mechanism. It appears that several phenomena may be at play simultaneously. This hypothesis may explain another persisting controversial issue related to the existence and the role of the acoustic feedback mechanism.

Lowson, Nash and McAlpine in their works argued that the feedback loop was not a necessary condition for the generation of tonal noise. They suggested that such mechanism if exists might be present about the separation bubble but this was never observed in acoustic spectra. Instead, more recent experiments by Kingan \& Pearse ${ }^{14}$ as well as DNS study by Desquesnes et $\mathrm{al}^{15}$ re-discovered the multi-tone spectrum of Arbey \& Bataille ${ }^{5}$ (Fig. 2) consistent with the feedback loop between the trailing edge and the onset points for T-S instability waves. Using this mechanism as a model for tonal frequency selection, Kingan \& Pearse ${ }^{14}$ employed stability analysis and XFOIL software to calculate T-S wave amplification rates. The obtained dominant frequencies compared reasonably well with results from the previously quoted experimental studies and somewhat less successfully with the empirical model of Brooks et al ${ }^{16}$. However, the prediction of frequencies could not be extended to prediction of the tone levels. As noted in Kingan \& Pearse ${ }^{14}$, those could not be easily related to the instability amplification in the boundary layer because of the nonlinear nature of the feedback mechanism. Such important connection has not been fully addressed to this day though it directly relates to the critical issue of boundary-layer excitation and dynamics induced by the acoustic feedback loop.

The current work re-visits the problem using complementary experimental and numerical studies investigating the presence of the flow-acoustic feedback interactions in NACA0012 airfoil in the transitional flow regimes. In the following discussion, we first describe the employed experimental and numerical approaches. Next, results of parametric experimental studies are discussed conducted both with clean and lowturbulence inflow, with boundary-layer tripping (on one or both sides) and without tripping, at different angles of attack, and for a range of flow speeds corresponding to 
$M=0.03 \ldots 0.15\left(R e_{c}=0.5 \times 10^{5} \ldots 3 \times 10^{5}\right)$. Finally, preliminary results of numerical 2D high-accuracy studies are provided focusing on selected regimes with uniform upstream flow conditions and investigating effect of Reynolds number on the boundary-layer statistical moments and airfoil frequency response signature.

\section{EXPERIMENTAL AND NUMERICAL APPROACHES}

\subsection{Experimental approach}

The work by Arbey \& Bataille $^{5}$ on the feedback-loop mechanism of tonal noise production associated with the acoustic scattering of amplified boundary-layer instabilities was based on a series of experiments conducted in an anechoic low-speed wind tunnel facility at Ecole Centrale de Lyon (ECL) (Fig. 4). The current experimental investigation employs the same facility to essentially re-visit the work of Arbey \& Bataille $^{5}$ at a more detailed level. The tonal signature of the low-speed NACA0012 airfoil is examined for a range of transitional flow regimes characterized by variable flow velocity, angle of attack, and unsteady inflow conditions to allow careful mapping of the regions of tonal production including effects of upstream unsteadiness.

Issues raised by Nash et $\mathrm{al}^{9}$ related to possible installation effects on acoustic feedback loop motivated the definition of a new design of the mounting device. The new configuration (Fig. 5) minimizes spurious reflections from the end-plate surfaces used in most experimental setups described in the literature and ensures that upstreampropagating sound from the trailing edge is the only mechanism of acoustic excitation

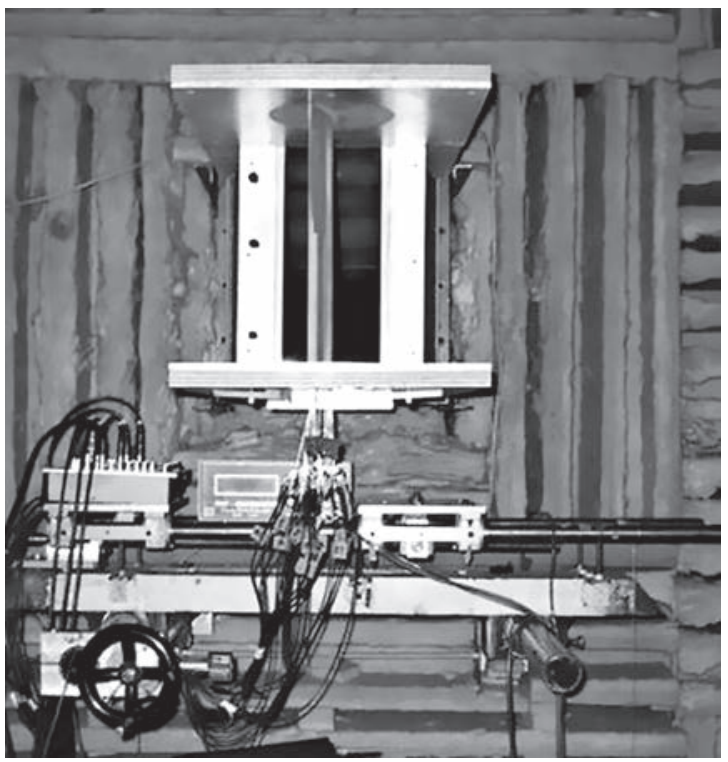

Figure 4: Picture of ECL facility showing the narrow nozzle flow and the horizontal end-plates. 


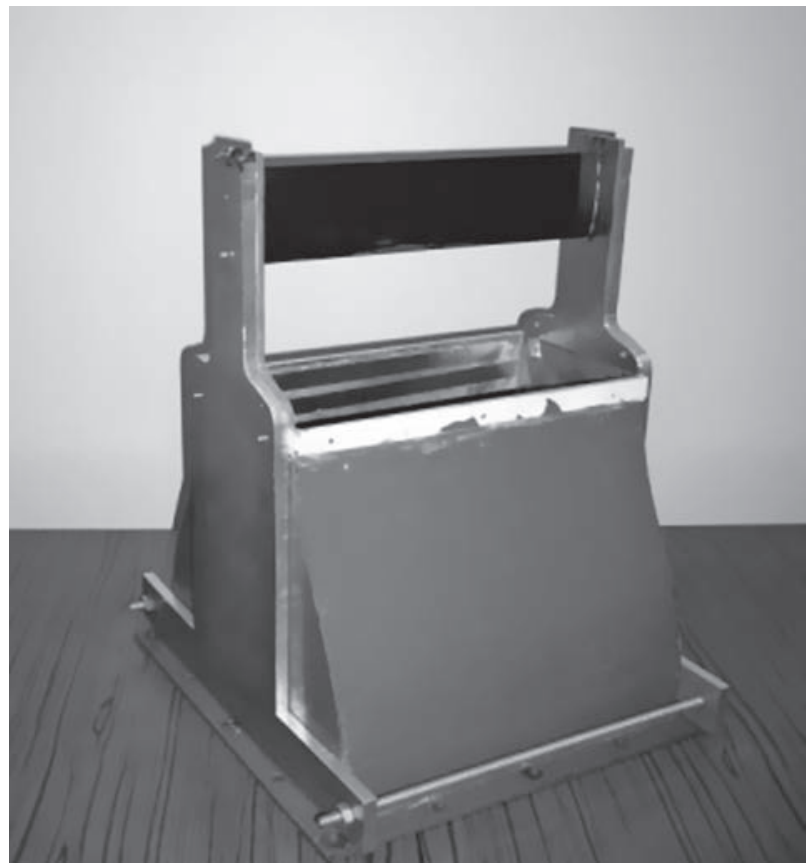

Figure 5: New mounting device minimizing installation effects.

for instabilities. This new design has been tested and confirmed that larger end plates produce no impact on the results. The ladder-type structure of the acoustic signature is thus believed intrinsic to the development and radiation of instability waves in the transitional flow. The only possible remaining installation effect is the jet-flow deflection due to lateral momentum injection associated with the mean lift as the airfoil is set at a non-zero angle of attack. However, this is not believed to significantly modify the underlying physics of the observed phenomena.

Far-field acoustic measurements performed in the mid-span plane using a single $1 / 2$ ' $\mathrm{B} \& \mathrm{~K}$ microphone on a rotating arm provide an overview of frequency-angle characterization and spectral directivity of the sound. To correlate acoustics with wallpressure fluctuations, an elaborate technique of measurements using remotemicrophone probes (RMPs) is employed. Such probes consist of capillary tubes embedded into the airfoil surface, perforated at the measuring end with enlargement outside the mock-up, and connected to laterally flush-mounted $1 /{ }_{4}^{\prime}$, B\&K microphones. The wall pressure at the pin-hole aperture of the probes excites acoustic waves propagating inside the capillary and measured by the microphones. The waves are damped along a long soft PVC tube closed at the opposite end in order to avoid reflections. Provided the response of the probe circuit is calibrated first for sound attenuation in the capillary, the wall pressure fluctuations can be measured at different locations on the surface by clusters of probes, with the microphones placed away from 
the measuring area to release obstruction constraints. The capillary diameter is $1 \mathrm{~mm}$ while the pin-hole diameter is $0.5 \mathrm{~mm}$ so the technology enables measurements in very thin mock-up parts with a typical threshold of $1.5 \mathrm{~mm}$, thus including a close vicinity of the trailing edge. Furthermore, the probes can be used to acquire either acoustic or unsteady aerodynamic responses and suitable from stable laminar to unstable turbulent boundary layer regimes. Other probe advantages include high achieved spatial resolution and the ability to acquire signals at very low levels. It must be noted that the installation of capillary tubes inside the airfoil results in spurious surface accidents which might have an effect on the transitional boundary layers. Therefore an additional mock-up without any instrumentation has been tested for comparison and reference, and some of the measurements have been carried out with two mock-ups of chord lengths $8 \mathrm{~cm}$ and $10 \mathrm{~cm}$. Note that the calibration of RMPs is carefully designed to correct effects of the capillary-tube viscous attenuation and multiple reflections of the waves in the varying cross-section tubes. This is achieved through complementary application of the analytical transfer function between the pin-hole and the remote microphone and a dedicated acoustic calibrator.

Additional measurements with a single Dantec hot-wire traversing probe installed on a minimally-intrusive L-shaped support complement pressure measurements with nearwake velocity profiles $2 \mathrm{~mm}$ downstream of the trailing edge. The extent of the hot-wire probe at the measuring location is about $3 \mathrm{~mm}$. Furthermore, the steady-state pressure coefficient is checked by simply connecting the RMP tubes to a manometer, while flow visualizations using distemper fluid are conducted to confirm laminar, turbulent and/or separated boundary layer regions and particularly identify the presence of laminar separation bubbles in each test case.

\subsection{Numerical approach}

High-accuracy numerical simulations are conducted using a modified version of Implicit Large Eddy Simulation (ILES) solver FDL3DI (Visbal \& Gaitonde ${ }^{17}$ ). The following features of the original code are particularly beneficial for the current study:

- Implicit time marching algorithms (up to $4^{\text {th }}$-oder accurate) are well suited for the low-Re wall-bounded flows.

- High-order spatial accuracy (up to $6^{\text {th }}$-order accurate) is achieved by use of implicit compact finite-difference schemes, thus making LES resolution attainable with minimum computational expense.

- Robustness is achieved through a low-pass Pade-type non-dispersive spatial filter that regularizes the solution in flow regions where the computational mesh is not sufficient to fully resolve the smallest scales. Note that the governing equations are represented in the original unfiltered form used unchanged in laminar, transitional or fully turbulent regions of the flow. The highly efficient Implicit LES (ILES) procedure employs the high-order filter operator in lieu of the standard SGS and heat flux terms, with the filter selectively damping the evolving poorly-resolved highfrequency content of the solution.

- Overset grid technique is adopted for geometrically complex configurations, with high-order interpolation maintaining spatial accuracy at overlapping mesh interfaces. 
All parameters in the code are non-dimensionalized with respect to the flow density, upstream mean velocity, and the characteristic length (e.g., an airfoil chord $c$ ). The original version of the code was tested and validated against numerous benchmark problems (e.g., in the works by Visbal \& Rizzetta ${ }^{18}$ and Visbal et al ${ }^{19,20}$ ). The modified version employs successfully tested capability for high-fidelity analysis of unsteady flow-structure interactions involving descriptions of non-uniform, vortical upstream flowfields and dynamic airfoil capability including two-degrees-of-freedom aeroelastic structural response (Refs 21-25). The code employs an efficient MPI parallelization successfully utilized in the cited works.

To compare numerical solutions with previous numerical and present experimental results for an airfoil with the clean upstream flow conditions, the current numerical study employs a $643 \times 395 \times 3$ O-mesh generated about NACA0012 airfoil (Fig. 6) and efficiently partitioned into sets of overlapped blocks assigned to different processors during parallel implementations. The mesh is carefully clustered near the airfoil surface to achieve the wall-normal and wall-tangent mesh sizes of $\Delta y / c=5 \times 10^{-5}$ and $\Delta \mathrm{x} / \mathrm{c}=10^{-3}$. In terms of the wall units $\mathrm{y}_{\mathrm{w}}{ }^{+} / \mathrm{c}=6.2 \times 10^{-5}$ estimated for the characteristic flow condition with $\mathrm{M}=0.1$ and $\mathrm{Re}=2 \times 10^{5}$, such grid refinement corresponds to the non-dimensional values of $\Delta \mathrm{y}^{+} \approx 1$ and $\Delta \mathrm{x}^{+}=20$, with 12 grid points clustered in the region $0<\mathrm{y}^{+}<10$. For 3D simulations, such grid parameters would correspond to a high-resolution LES according to estimates in Wagner et $\mathrm{al}^{26}$ (p.209). The current grid is also finer compared with the mesh employed in a similar DNS study by Desquesnes et al ${ }^{15}$ conducted using the mesh with $\Delta \mathrm{y} / \mathrm{c}=3.8 \times 10^{-4}$ and $\Delta \mathrm{x} / \mathrm{c}=6 \times 10^{-3}$.

Note that the current paper reports on the results of $2 \mathrm{D}$ simulations that are compared against corresponding numerical solutions of Desquesnes et $\mathrm{al}^{15}$ and present experimental data. Such approach is based on the assumption that the flow regimes remain primarily laminar (with possible separation zones) but may start to exhibit transitional features.
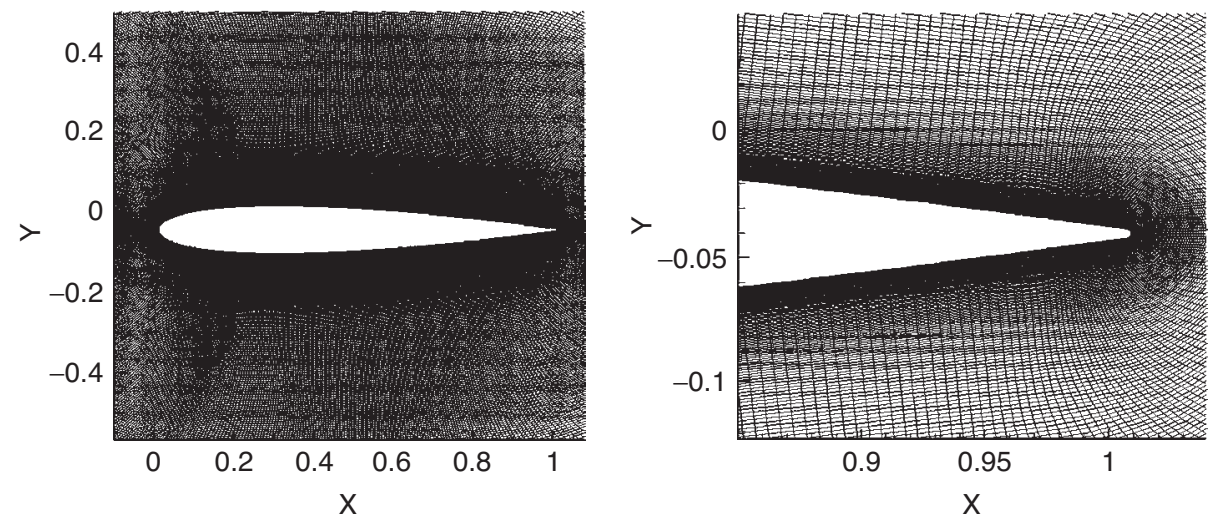

Figure 6: $\quad$ Mesh generated around NACA0012 airfoil (left); mesh details near the trailing edge (right). 
The developed numerical method appears well-suited for the analysis of the effect of impinging disturbances on the airfoil flow-acoustic interactions. Although the current work does not address such impact in comparison with presented experimental results, the future study will employ a recently developed approach to introduce convected flow disturbances in numerical simulations through a momentum source region located upstream of the airfoil. The proposed method ${ }^{27}$ is a $3 \mathrm{D}$ extension of the $2 \mathrm{D}$ gust source model previously developed by Golubev et $\mathrm{al}^{21}$. The general approach to produce synthetic turbulent field employs random flow generation (RFG) technique of Smirnov et $\mathrm{al}^{28}$ recently advanced by Huang et $\mathrm{al}^{29}$ to match von Karman turbulence spectrum. The proper superposition of gust harmonics in the new momentum source module follows RFG procedures to effectively synthesize turbulence field with given integral characteristics. Corresponding parameters will be extracted from the current experimental measurements with turbulence grids.

\section{EXPERIMENTAL RESULTS}

\subsection{Results for uniform upstream flow}

3.1. Acoustic Frequency-Directivity Maps

A series of experiments have been designed to carefully examine the ladder-type structure of acoustic tones for a range of transitional airfoil flow conditions. Preliminary results obtained in this work using the anechoic wind tunnel facility of Ecole Centrale de Lyon clearly confirm the existence of the ladder-type structure observed by Arbey \& Bataille ${ }^{5}$. The tones disappear when the flow transitions to fully turbulent regime. However, as pointed out by McAlpine et $\mathrm{al}^{11}$, the onset of turbulence has to be far enough upstream of the trailing edge to have a sufficient distance to destroy coherent structures of the boundary-layer waves and thus completely remove the tonal noise content.

Initial tests were conducted with NACA0012 rectangular airfoil sections of $8 \mathrm{~cm}$ and $10 \mathrm{~cm}$ chords and of $30 \mathrm{~cm}$ span (aspect ratio of nearly 3 ), both in clean flow and with controlled inflow disturbance, with boundary-layer tripping (on one or both sides) and without tripping, at different angles of attack, and for a range of flow speeds corresponding to $M=0.03 \ldots 0.15\left(\operatorname{Re}_{c}=0.5 \times 10^{5} \ldots 3 \times 10^{5}\right)$. Tripping a transitional boundary layer is known to force transition to turbulence and avoid the onset of coherent instabilities. Some tests have been made here using medical tape on one side of the airfoil in order to let a natural laminar boundary layer develop on the other side. It has also been verified that tripping both sides only produces broadband noise at a lower level. The one-side tripping was suggested by results from the recent DNS study of Desquesnes et $\mathrm{al}^{15}$ who proposed another view on the multi-tone acoustic response involving interaction of the main and the secondary feedback loops on both sides of the airfoil. However, the present results demonstrate that the ladder-type acoustic tonal structure may reveal itself without such interaction. This is emphasized in Fig.7 where the noise amplitude measured at $90^{\circ}$ to the flow and at $2 \mathrm{~m}$ in the mid-span plane is plotted as a function of frequency and flow speed. The results are obtained for the one-side tripped airfoil of $8 \mathrm{~cm}$ 

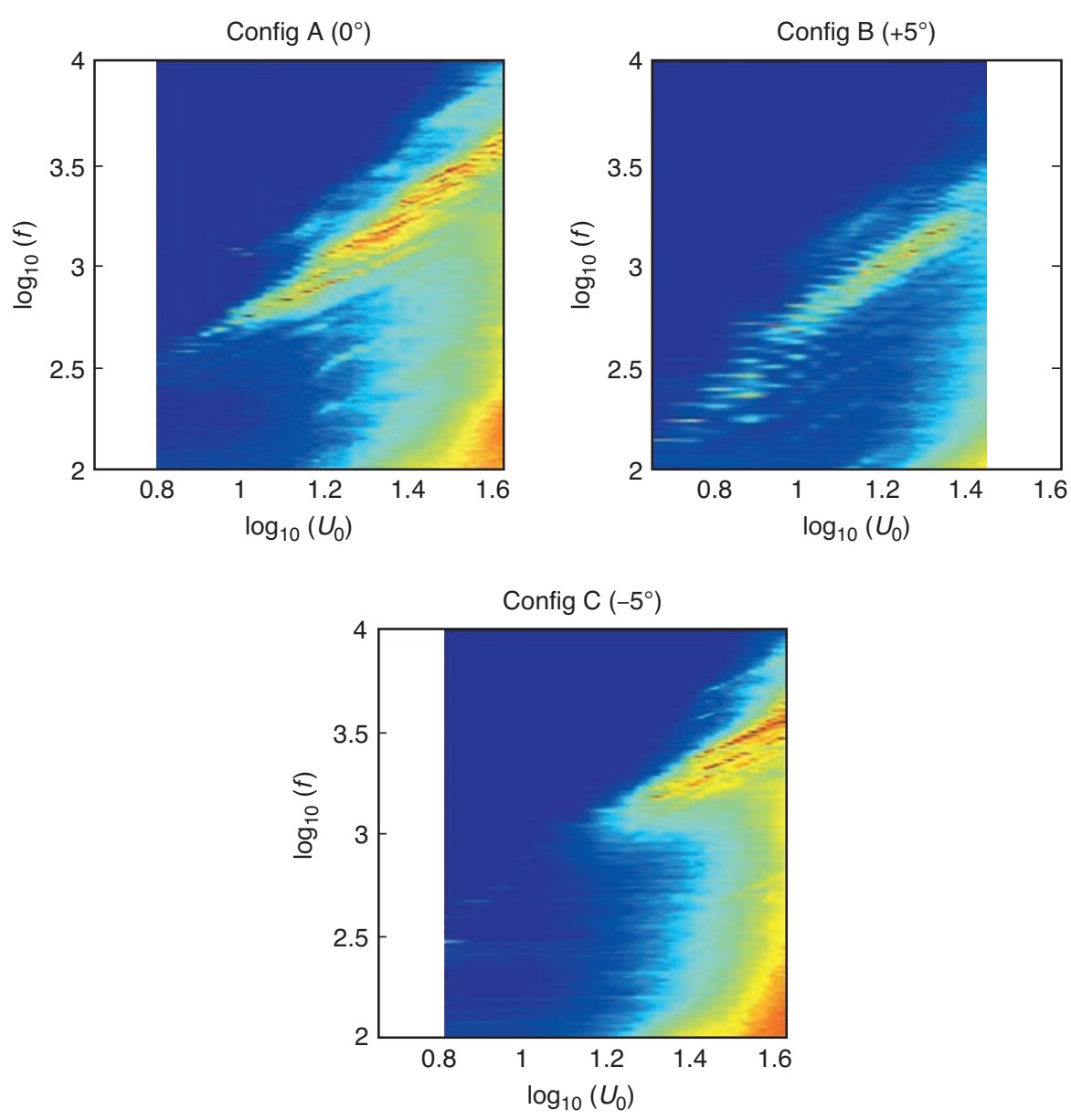

Figure 7: Frequency versus flow-speed charts of airfoil tonal noise. One-side tripped NACA0012, $8 \mathrm{~cm}$ chord length, tripping strip on the pressure side $\left(+5^{\circ}\right)$ and on the suction side $\left(-5^{\circ}\right)$.

chord in the mounting configuration of Fig. 5, at geometrical angles of attack $-5^{\circ}$, $0^{\circ}$ and $+5^{\circ}$. Apart from the low-frequency contribution at the bottom right corner of each color map, the tonal signature is clearly observed in each case, with different amplitudes and frequency ranges. The global oblique trace follows $f_{s} \sim U^{1.5}$ (yellow patterns) though the trend is less pronounced for Configuration B. Superimposed in red are the lines (rungs) corresponding to tones with $f \sim U^{0.8} \ldots 0.85$. Overall, multiple tones are observed at a given flow speed, as shown in Fig. 7 and further in Fig. 8.

The tripping ensures that one of the boundary layers is turbulent, which is partly responsible for the low-frequency content of the spectrum. The tonal noise, in contrast, 

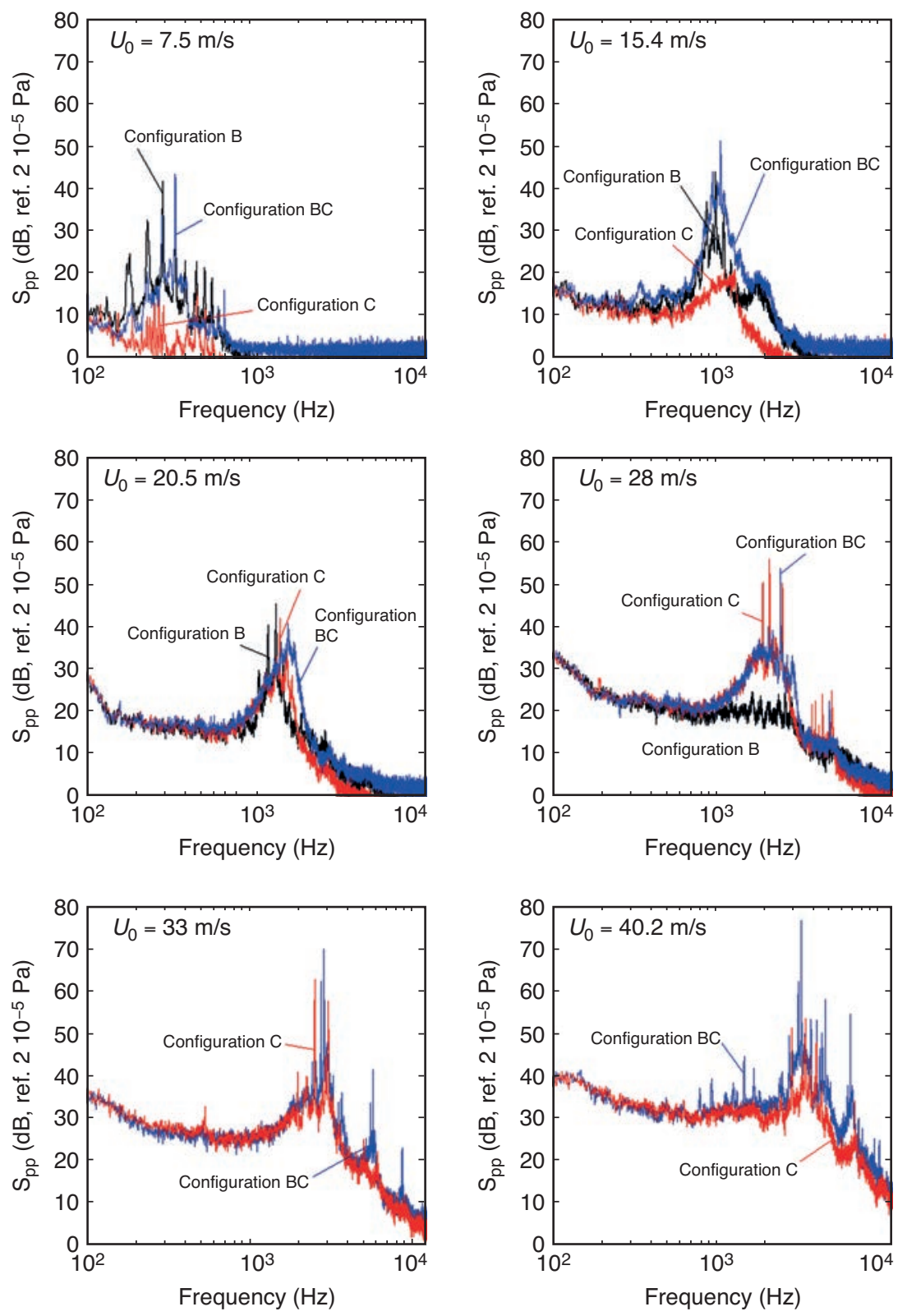

Figure 8: $\quad$ Typical sound spectra measured at $5^{\circ}$ angle of attack, at various flow speeds. B: tripping on pressure side, $\mathrm{C}$ : tripping on suction side, $\mathrm{BC}$ : no tripping. 
is associated with the laminar instabilities. Interestingly, the clearest ladder-type structure is found at zero angle of attack (Configuration A) and is close to observations by Arbey \& Bataille ${ }^{5}$. In Configuration B, the tripping is on the airfoil pressure side and the sound originates from instabilities amplifying along the suction side. The tones are less numerous and at much lower level but, in contrast, they can be detected at lower flow speeds. In Configuration C, the tripping is on the suction side and visualizations suggest that flow separates over a large part of the pressure-side boundary layer. Multiple tones are also detected but with a less organized structure. Moreover, their occurrence requires significantly higher flow speed.

Based on the obtained data, it is our current view that two separate phenomena are, in fact, at play in the ladder-type structure of acoustic tones. They correspond to the rungs with $f \sim U^{0.8}$ related to the amplified instability wave trailing-edge scattering, and the effectively produced vortex shedding corresponding to the dominant frequencies of each rung scaled with $f_{s} \sim U^{1.5}$. The exact conditions for the observed jumps between the rungs remain unknown and will be the subject of future investigations using highfidelity numerical analysis. It may also be suggested that the experiments of Lowson, Nash and McAlpine were able to observe just a single rung in their data, with some mechanism responsible for preventing the jump occurrence.

\subsubsection{Effect of one-side tripping}

Typical acoustic spectra extracted from the maps of Fig.7 at the geometrical angle of attack of $5^{\circ}$ are shown in Fig. 8 for a closer comparison. The configuration BC stands for the no-tripping case. At high flow speeds, the configuration $\mathrm{B}$ does not produce significant tonal noise and the $\mathrm{C}$ and $\mathrm{BC}$ correspond to similar acoustic signatures. More precisely, the main humps attributed to the primary radiation are similar and differences are seen in the superimposed tones. Tripping only one side or the other one probably induces significant changes in the aerodynamic performances of the airfoil; furthermore, changes in the angle of attack induce small positioning errors which T-S waves are very sensitive to. Despite this, it can be concluded that the sound-generating mechanism takes place on the pressure side at flow speeds starting from typically $28 \mathrm{~m} / \mathrm{s}$.

In contrast, at the lowest flow speeds, the signature of configuration $\mathrm{C}$ is less pronounced whereas configurations $\mathrm{B}$ and $\mathrm{BC}$ behave in a comparable way. This suggests that the sound and particularly the multiple tones are related to the processes on the suction side in such cases.

\subsection{Wall-Pressure Measurements}

The NACA-0012 airfoil of $10 \mathrm{~cm}$ chord length is implemented with a series of remotemicrophone probes (RMPs) which allow measuring both the wall-pressure fluctuations and the steady-state pressure coefficient. The latter is the key information at non-zero angle of attack when the measurements are dedicated to the comparison with numerical simulations. Indeed, the jet issuing from the nozzle of the wind-tunnel is deflected by the lateral momentum injection associated with the mean lift on the airfoil. Even though correction formulae have been provided to correct the geometrical angle of attack in the 


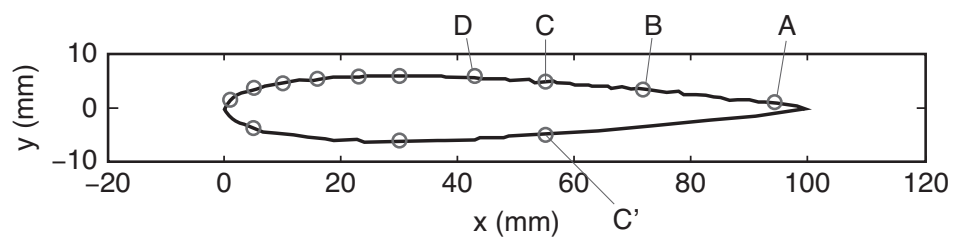

Figure 9: Locations of the RMPs on the NACA-0012 mock-up (red symbols) and reference points for the analysis of wall-pressure fluctuations.

wind tunnel $\alpha_{\mathrm{g}}$ for the equivalent effective angle of attack in infinite stream, the pressure coefficient has been shown to provide a more reliable information, as pointed out by Moreau et $\mathrm{al}^{30}$. This is essentially because the static pressure gradient along the surface determines the development of the boundary layers. Therefore, the pressure coefficient has been measured for both the zero angle of attack and the geometrical angle $\alpha_{\mathrm{g}}=5^{\circ}$. The instrumented mock-up has a larger number of probes on one side but both sides can be used for this characterization, provided that the airfoil is set at negative or positive incidence.

The locations of the remote-microphone probes used for the wall-pressure measurements are pointed as red symbols in Fig.9. The measured steady-state pressure coefficients are included in the comparison with numerical predictions below. Furthermore, the acquisition points of the wall-pressure fluctuations discussed further on are indicated by capital letters A, B, C, D and C'.

The unsteady pressure measurements have been performed in the same flow conditions at the flow speed of $25 \mathrm{~m} / \mathrm{s}$. The installation of the capillary tubes of the RMPs just beneath the surface may induce some micro-disturbances. Therefore, as the angle of attack is set negative or positive with the same values, discrepancies are seen either because of small positioning errors or differently developing instability waves due to surface imperfection. Despite this shortcoming, the overall relevance of the results is taken for granted.

At zero angle of attack, $C$ and $C$ ' locations should measure the same wall-pressure spectra. This is not exactly the case as shown in Fig. 10 where the spectra are labelled $\mathrm{C}$ and $\mathrm{C}$ (other) in green and black. Furthermore, the signals measured at $\mathrm{C}$ and $\mathrm{D}$ are very similar. In view of their similarity of spectral shapes with the far-field sound, the signals at points $\mathrm{B}, \mathrm{C}$ and $\mathrm{D}$ are either of acoustic nature or correspond to processes which have the same degree of coherence as the acoustic waves. The sound at B may be larger because of the closer vicinity to the trailing edge where the sources are located.

In contrast, the signal at point $\mathrm{A}$ is more likely to be of aerodynamic nature because of its different shape and the very high level.

The asymmetrical behaviour due to the difficulty of ensuring perfect positioning or the surface smoothness is emphasized in Fig.11, where the spectra at points $\mathrm{C}$ and $\mathrm{C}$ ' for the angles $+5^{\circ}$ and $-5^{\circ}$ do not coincide despite the expected equivalence of flows. In contrast, the far-field sound spectra are close to each other except for a couple of 


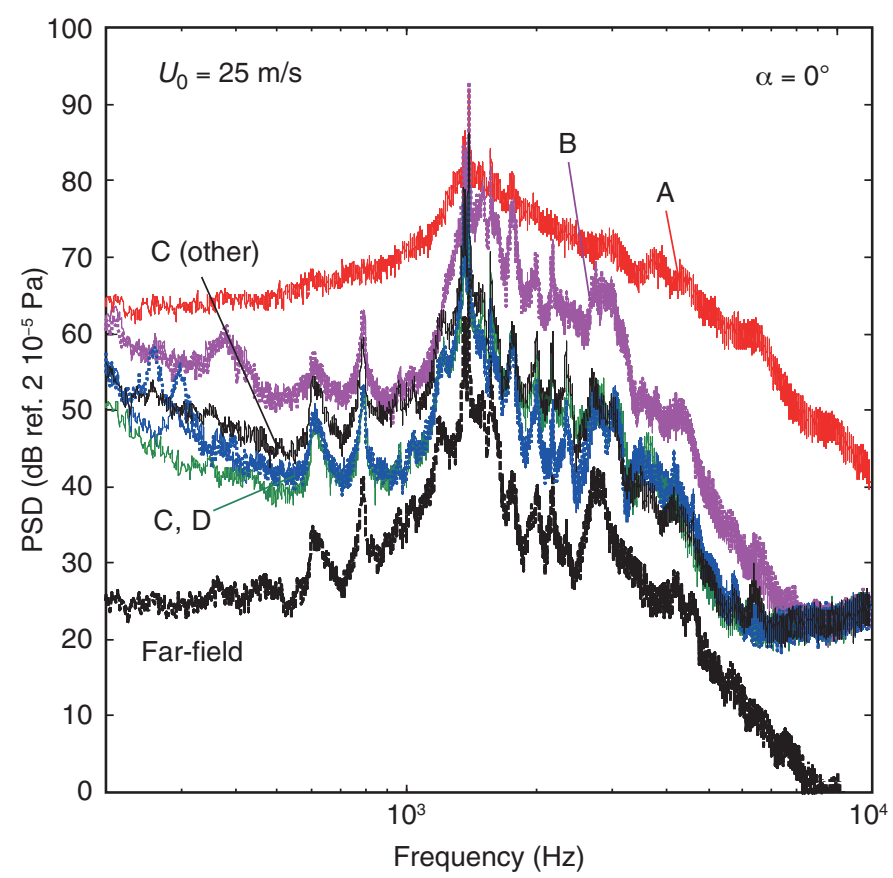

Figure 10: Far-field sound and wall-pressure spectra at zero angle of attack.

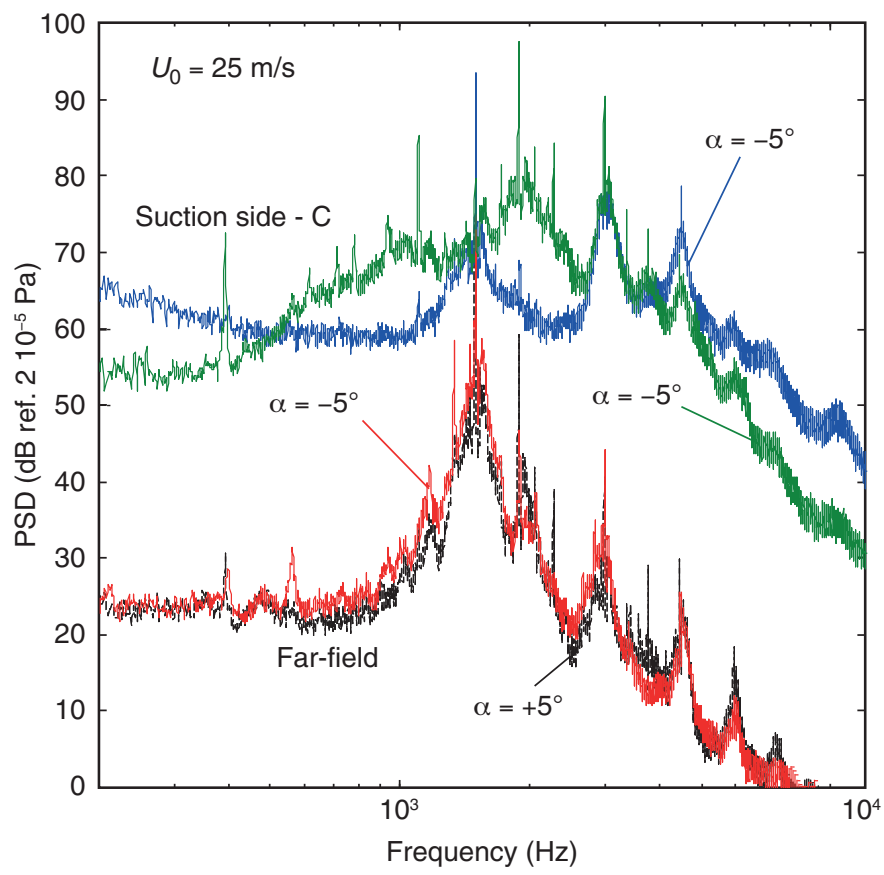

Figure 11: Compared spectra in the far-field and at point $\mathrm{C}(\mathrm{N})$ for positive and negative angles of $5^{\circ}$. 
tones. This might indicate that what happens at location $\mathrm{C}$ on the suction side is not the most essential aspect for sound radiation.

Setting the angle of attack to $5^{\circ}$ with the RMPs A to D on the suction side, and getting the signal at location $\mathrm{C}\left(\mathrm{C}^{\prime}\right)$ on the opposite side, provides the results of Fig. 12. Again, the pressure side signal at $\mathrm{C}$ is similar to the far-field spectrum, whereas all signals from the suction side are of high amplitude and probably of aerodynamic nature. The corresponding results obtained when setting the airfoil at $5^{\circ}$ with most RMPs on the pressure side are shown in Fig.13. As suspected before, the signals from RMPs B, $\mathrm{C}$ and $\mathrm{D}$ are expectedly of acoustic nature because of their similarity with the far-field sound spectrum. Another possible explanation is their coherence relationship with the acoustic waves, which would trigger similar envelopes. In contrast, the signal at point $\mathrm{A}$ is of much higher amplitude, with the same order of magnitude at its maximum as the signal from RMP C on the suction side.

\subsection{Results for turbulent upstream flow}

\subsubsection{Acoustic Far-Field Measurements}

Incident turbulence forces random oscillations on the airfoil surface and accelerates transition in the boundary layers. At high turbulence rates, this is known to produce turbulence-interaction noise also referred to as leading-edge noise instead of the selfnoise or trailing-edge noise observed in clean flow. But intermediate regimes are also expected for small-scale and low-intensity turbulence. This is why a series of measurements has been made including a turbulence grid upstream of the airfoil, in the nozzle duct. The grid mesh is of $1 \mathrm{~cm}$ and the diameter of the mesh wires is $1.5 \mathrm{~mm}$. The grid is installed 60 mesh sizes upstream of the airfoil, and the nozzle contraction rate is 2 , from 30 by $30 \mathrm{~cm}$ to 30 by $15 \mathrm{~cm}$ at the exit.

The generated turbulence has been characterized by single-hot wire measurements made at the location of the airfoil leading edge but with the airfoil removed. The results are synthesized in Fig. 14. The residual turbulence of the wind tunnel featured by the black-line spectrum is at a very low level and defines the clean-flow conditions for which the TS wave radiation exhibits multiple tones. The turbulence spectrum measured in the presence of the grid is plotted in red and fit with the homogeneous and isotropic von Kármán model (dashed black line) accounting for an additional Gaussian attenuation to reproduce the drop towards the Kolmogorov scale (cut-off frequency around $9500 \mathrm{~Hz}$ ). The corresponding integral length scale is $3.5 \mathrm{~mm}$ and the rms velocity is of $0.2 \mathrm{~m} / \mathrm{s}(0.8 \%$ turbulence rate $)$. It has been verified that the background noise measured with the grid installed remains well below the airfoil noise of interest.

As shown next from the maps in Fig. 15, the effect of upstream turbulence is obviously to suppress all tones associated with acoustic feedback and corresponding to the 0.85 -power ladder-type structure. But the main trace of the hump is preserved and still follows the 1.5-power law, plotted at the same level as the black dashed-line in all plots. The tests have been repeated with airfoils of chord lengths $8 \mathrm{~cm}$ and $10 \mathrm{~cm}$, producing the same observations. It is concluded that the main dynamics of the transitional boundary layer is not fundamentally modified by the free-stream 


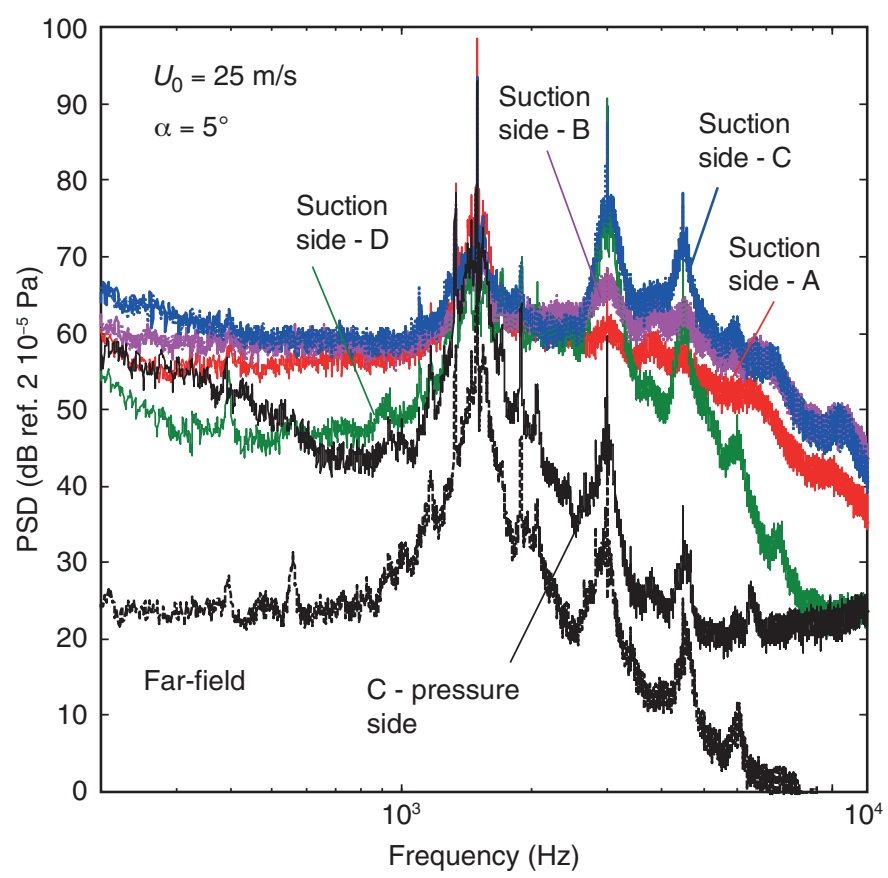

Figure 12: Far-field sound and wall-pressure spectra at $5^{\circ}$ angle of attack with RMPs $\mathrm{A}, \mathrm{B}, \mathrm{C}, \mathrm{D}$ on the suction side.

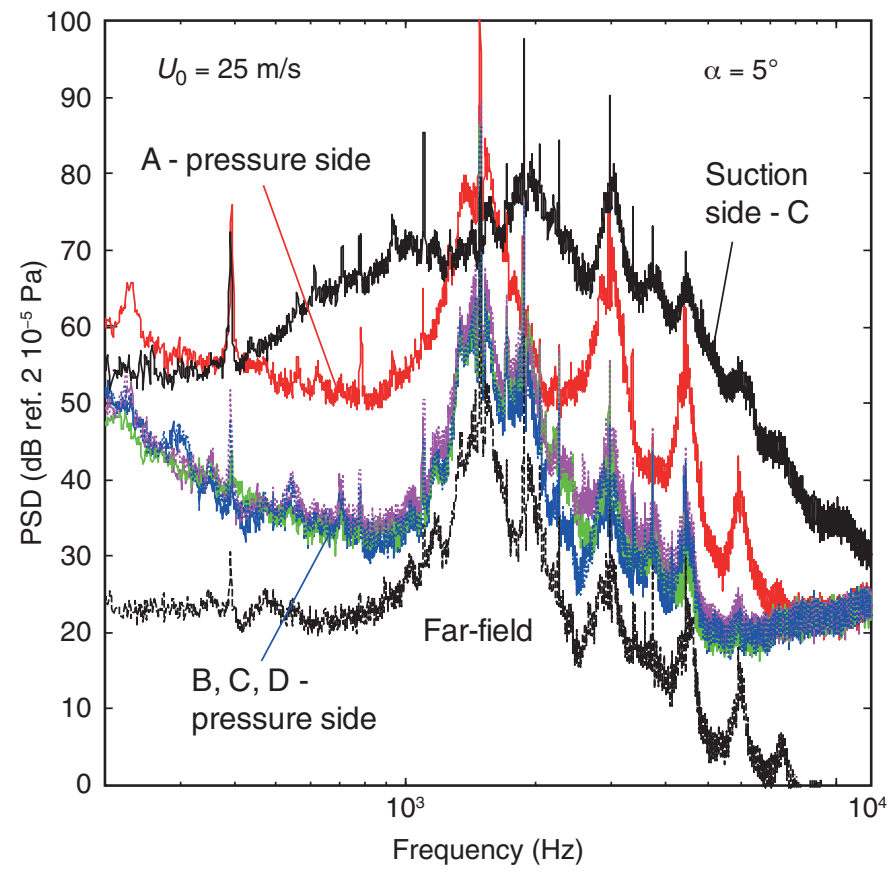

Figure 13: Far-field sound and wall-pressure spectra at $5^{\circ}$ angle of attack with RMPs A, B, C, D on the pressure side. 


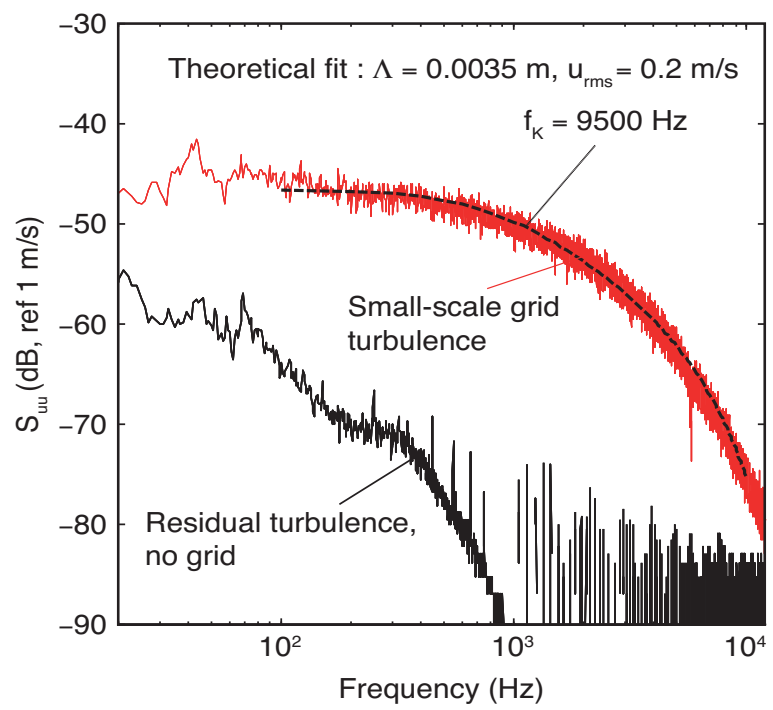

Figure 14: Streamwise turbulence spectra measured at the location of the leading edge (airfoil removed). Residual turbulence in clean-flow configuration (black) and grid-generated low-level turbulence (red). Mean flow speed $25 \mathrm{~m} / \mathrm{s}$.

turbulence, whereas its resonant receptivity to acoustic waves might be deactivated. Interestingly, the signature is at slightly higher frequencies for the smaller airfoil, especially at $0^{\circ}$ angle of attack, as expected.

\subsubsection{Near-Wake Characterization}

Scanning the near-wake velocity profiles is another way to investigate the flow features of interest. It also provides reference data that can be further compared to numerical simulations for validation purposes. The measurements reported in Fig. 16 are made at $25 \mathrm{~m} / \mathrm{s}$ with the $10 \mathrm{~cm}$ chord airfoil, both with clean inflow and with the small-scale turbulence produced by the grid inserted upstream of the nozzle. In both inflow conditions, the velocity profiles in the wakes are measured $2 \mathrm{~mm}$ downstream of the trailing edge along a path perpendicular to the incident flow direction, at both zero angle of attack and at the angle $5^{\circ}$. In the latter case, the profile path is not normal to the wake axis. Both sides of the airfoil are untripped. The origin is taken where the probe is exactly facing the streamwise continuation of the trailing edge.

At zero geometrical angle of attack, the wake is nearly symmetric as expected, with a weak imbalance in the clean-flow configuration, more pronounced in the rms-velocity distribution. The symmetry is recovered in the presence of turbulence. At $5^{\circ}$ angle of attack, the expected asymmetric wake is found, with thinner and thicker boundary-layer continuations from the pressure-side and suction-side, respectively. A slightly thinner wake is measured in clean inflow conditions. In general, introducing 



Figure 15: Frequency versus flow-speed charts for a symmetrical NACA-0012 airfoil of $10 \mathrm{~cm}$ (top) and $8 \mathrm{~cm}$ (bottom) chord length set at $0^{\circ}$ and $5^{\circ}$ angles of attack in low-intensity upstream turbulence. No tripping.

weak free-stream turbulence only produces minor changes in the present investigation; essentially, the main features of the flow remain the same.

\subsubsection{Hot-Wire Measurements}

The spectrum of the streamwise velocity fluctuations has been measured with the traversing hot-wire probe at 7 locations indicated as blue symbols in Fig. 16. The results for the angles of attack $0^{\circ}$ and $5^{\circ}$ with and without the turbulence grid installed are gathered in Fig. 17. The values of the transverse coordinates y of the symbols are indicated on the plots.

The results in the presence of the upstream turbulence grid are reported on the righthand side plots. At $0^{\circ}$ angle of attack the spectral envelope is broadband rather than showing evidence of any spectral signature associated with instability waves. In 

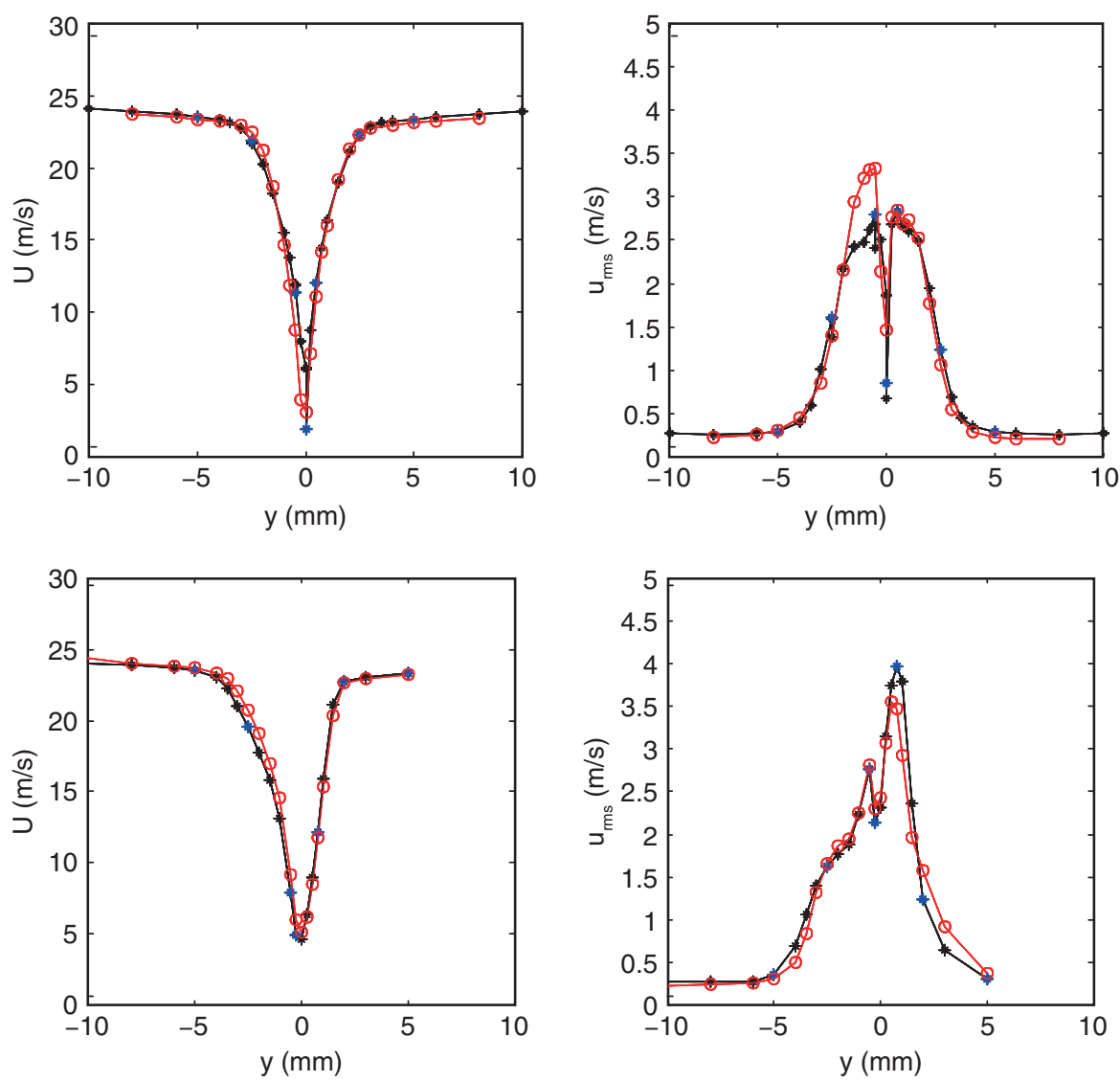

Figure 16: Near-wake velocity profiles at zero angle of attack (top) and $5^{\circ}$ (bottom), with (black) and without (red) upstream small-scale turbulence. Mean velocity on the left and turbulent rms velocity on the right. Oncoming flow speed $25 \mathrm{~m} / \mathrm{s}$. Blue symbols stand for locations of measured spectra.

contrast, a clear hump is seen at $5^{\circ}$, except at $\mathrm{y}=-5 \mathrm{~mm}$ just before entering the wake suction side, and at $\mathrm{y}=-0.25 \mathrm{~mm}$ corresponding to the wake centerline.

\section{NUMERICAL RESULTS}

\subsection{Validation study}

In their 2D DNS study of the trailing-edge noise radiated by the laminar NACA0012 airfoil, Desquesnes et $\mathrm{al}^{15}$ focused on the tones-producing flow regime with $\mathrm{M}=0.1$ and $\operatorname{Re}=2 \times 10^{5}$ for the airfoil with the chord of $c=0.3 \mathrm{~m}$ installed at the angle of attack $\alpha=2$ deg. Such flow configuration is examined first in the current study for comparison and validation of the numerical $2 \mathrm{D}$ approach. It should be noted that, to the best of the authors' knowledge, high-fidelity 3D numerical studies have not been previously attempted but such study is currently implemented and will be reported in the future work. 



Figure 17: Streamwise turbulence spectra measured at various locations by a traversing probe. Distance from the trailing edge $2 \mathrm{~mm}$. Angles of attack $0^{\circ}$ (top plots) and $5^{\circ}$ (bottom plots). Clean inflow (left) versus gridgenerated turbulence (right). The values of the lateral coordinate y are indicated.

Similar to Ref. 15, the physical time step of $0.3625 \times 10^{-6} \mathrm{sec}$ (corresponding to the non-dimensional value of $\Delta \mathrm{t}_{1}=4.19 \times 10^{-5}$ ) is adopted in the numerical simulations. The steady-state flow condition is reached after 200,000 steps and reveals a distorted boundary-layer region on the suction side in Fig. 18. The acoustic and near-field unsteady flow data was then collected with the sampling rate of $62.7 \mathrm{kHz}$ (i.e., recording every $44^{\text {th }}$ step). Over 8,000 samples were collected in Run 1 to obtain to frequency resolution of $\Delta \mathrm{f}_{1}=7.65 \mathrm{~Hz}$. To examine the effect of the time resolution, a separate computation (Run 2) was conducted with the twice reduced time step $\left(\Delta \mathrm{t}_{2}=\right.$ $0.5 \Delta \mathrm{t}_{1}$ ) while keeping the same sampling rate. Over 16,000 samples were collected for a total period of nearly $0.26 \mathrm{sec}$ after reaching the steady-state flow condition, thus 


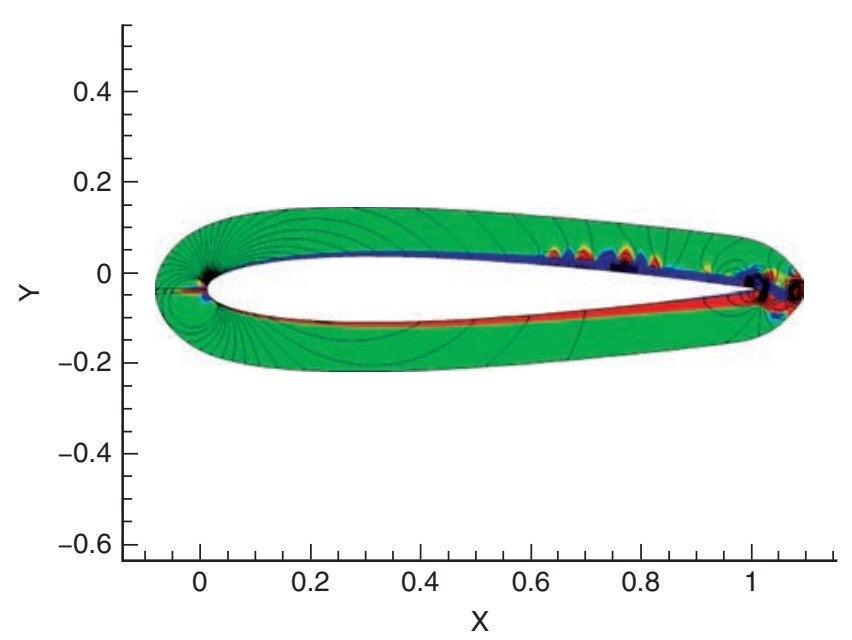

Figure 18: Vorticity (colored) and pressure contours.

Table 1. Comparison of RMS pressure levels (dB) at six points.

\begin{tabular}{lccc} 
& Run 1 & Run 2 & Ref. 15 \\
\hline Point 1 & 113.3 & 114.0 & 112.2 \\
Point 2 & 111.0 & 112.0 & 108.6 \\
Point 3 & 108.0 & 108.8 & 104.8 \\
Point 4 & 112.9 & 113.3 & 112.3 \\
Point 5 & 110.3 & 110.9 & 108.7 \\
Point 6 & 108.0 & 108.7 & 104.9
\end{tabular}

achieving the minimum frequency resolution of $\Delta \mathrm{f}_{2}=3.83 \mathrm{~Hz}$. Table 1 compares the RMS pressure $\mathrm{dB}$ levels obtained based on the two collected sets of data for six "virtual probes" of Ref. 14 located 0.5, 1 and 2 chords above (Points 1, 2 and 3) and below (Points 4, 5 and 6) the airfoil trailing edge. For Points 1 and 4 closest to the airfoil, the discrepancies between different runs and the results of Desquesnes et al ${ }^{15}$ is within 1-2 $\mathrm{dB}$. The latter results (obtained with $\Delta \mathrm{f}=1.84 \mathrm{~Hz}$ ) show faster decay of the acoustic pressure levels with increasing distance from the trailing edge, which could be related to the deteriorating grid resolution of the stretched mesh in one of the simulations.

The rest of the validation analysis is focused on Point 1 . The acoustic pressure spectra based on the two runs with different time steps and frequency resolutions reveal an overall similar distribution in Fig. 19(a) which appears also similar in shape but notably higher in the broadband level compared to the result from Ref. 15 shown in Fig. 19(b). However, the primary focus here is on the spectral peaks which are assumed to manifest the presence of the acoustic feedback loop with multiple tones corresponding to the selected frequencies of the amplified and scattered T-S waves. They are, in fact, 
(a)

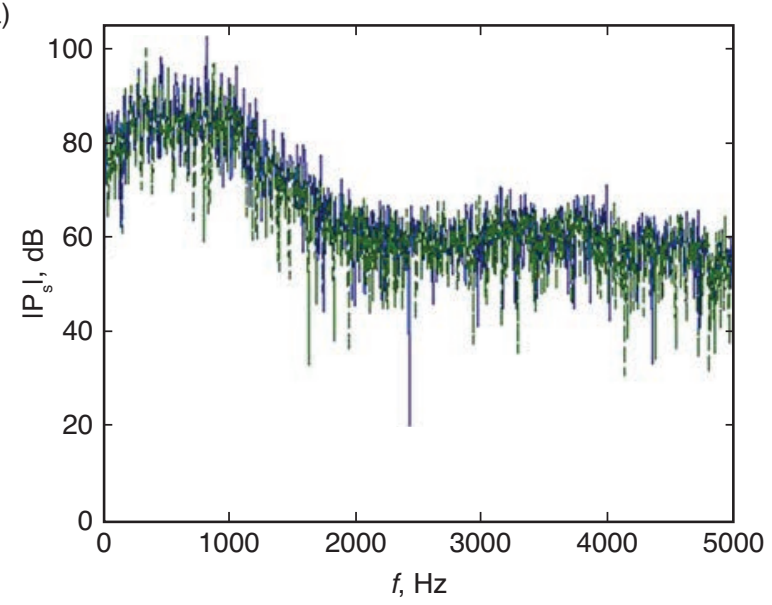

(b)

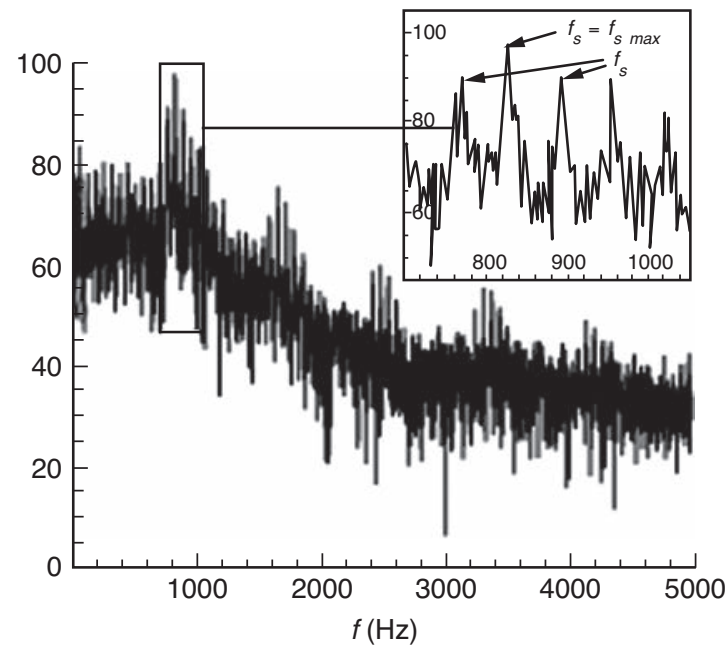

Figure 19: $\quad$ Pressure spectrum at Point 1: (a) Results from Runs 1 (solid blue) and 2 (dashed green), (b) Ref.15.

present in both analyses but appear to be sensitive both to the simulation time step and the frequency resolution. This is evident from Fig. 20(a) which zooms on the region of dominant spectral peaks while comparing results for the two runs with different time steps but with the same frequency resolution $\Delta \mathrm{f}_{1}=7.65 \mathrm{~Hz}$. The peak frequency shifts from $842 \mathrm{~Hz}$ to $888 \mathrm{~Hz}$ (results from Ref. 15 indicate the peak at $830 \mathrm{~Hz}$ ). Comparison of results for Run 2 with the same reduced time step but for the frequency resolutions $\Delta \mathrm{f}_{1}=7.65 \mathrm{~Hz}$ and $\Delta \mathrm{f}_{2}=3.83 \mathrm{~Hz}$ just shows some redistribution of $\mathrm{dB}$ levels for the primary peaks in Fig. 20(b). However, the difference between the primary peaks appears nearly constant around $60 \mathrm{~Hz}$ which is close to the prediction of Desquesnes 
(a)

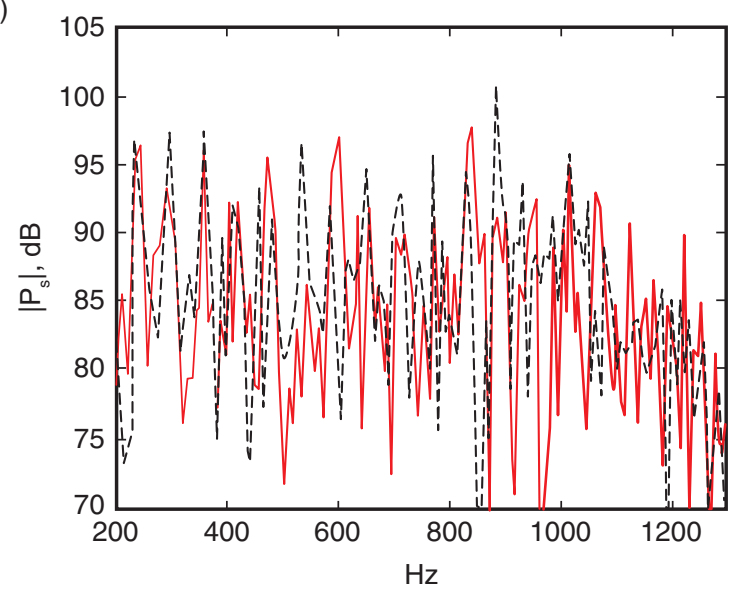

(b)

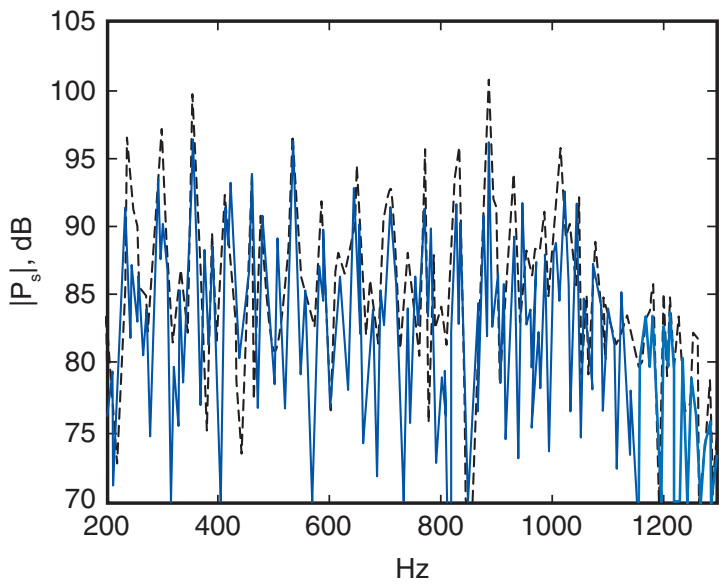

Figure 20: Details of pressure spectrum at Point 1 showing effect of (a) time step and (b) frequency resolution. Results obtained with $\Delta \mathrm{t}_{1}, \Delta \mathrm{f}_{1}$ (solid red); $\Delta \mathrm{t}_{2}, \Delta \mathrm{f}_{1}$ (dashed black), $\Delta \mathrm{t}_{2}, \Delta \mathrm{f}_{2}$ (solid blue).

et $\mathrm{a}^{15}$. Overall, despite certain sensitivity of the results of the spectral analysis, the overall response pattern remains similar in all the cases for the investigated range of numerical parameters. Note that high sensitivity of the unsteady data may be indicative of unaccounted, inherently 3D processes taking place in the transitional flow regimes. In a related sense, it also points to the highly nonlinear character of the physical processes involved in the flow-acoustic interactions. In experiments, the intermittency of the radiated tones induced by the scattered T-S waves was often witnessed and usually revealed using the spectrogram method wherein the total period of the signal is divided into a number of overlapping bands with the spectral analysis applied to 

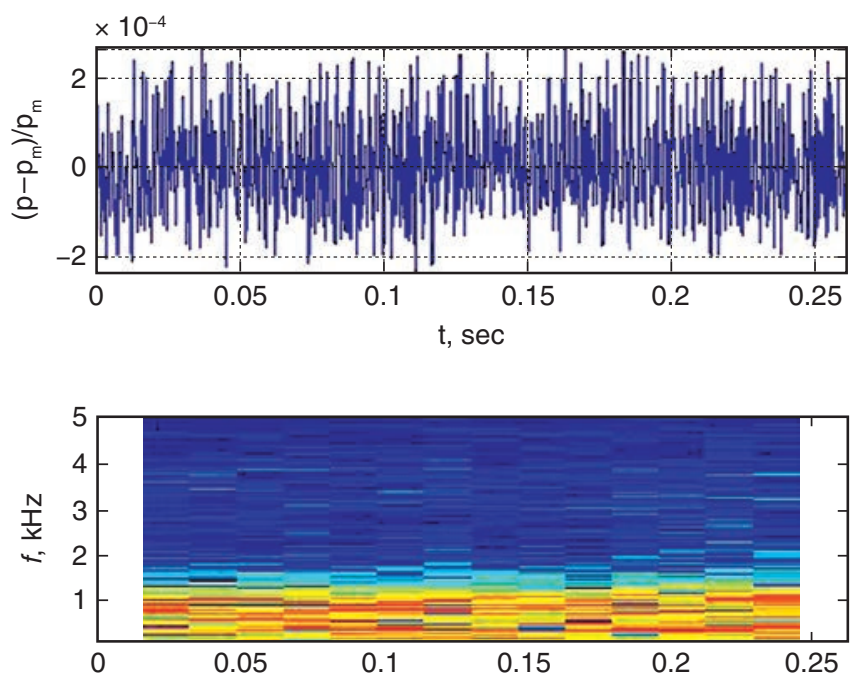

Figure 21: Spectrogram (bottom plot) of the pressure signal in Run 2 (top plot).

each band. Fig. 21 (bottom plot) illustrates the result of the method applied to the pressure signal extracted from Run 2 (top plot) using 15 bands with $50 \%$ overlap. The amplitudes of the present tones vary by color from deep blue to dark red. They appear to fluctuate with time although the main tonal frequency content remains unchanged. Desquesnes et $\mathrm{a}^{15}$ also realized the presence of the random component in the computed signal but proved the consistency of the primary tonal spectrum using the related periodogram method based on averaging the results of the Fourier transform applied to each time band.

Finally, in order to further examine the difference in RMS pressure levels shown in Table 1 between symmetrical (relative to the trailing edge) Points 1 and 4, Fig. 22(a) shows the corresponding pressure spectra obtained in Run 2. The peak values are nearly identical in the primary tonal region up to around $1 \mathrm{kHz}$ but the following decay is faster at Point 4 (below the trailing edge) which explains the overall RMS pressure level reduction by $0.7 \mathrm{~dB}$. Interestingly, the primary peak at $356 \mathrm{~Hz}$ was not detected in the study by Desquesnes et $\mathrm{al}^{15}$ who only observed the tonal zone surrounding the peak at $830 \mathrm{~Hz}$ (also present in Fig. 22(a)) and its higher harmonics. The slight asymmetry of the acoustic radiation above and below the airfoil is confirmed from the observed in Fig. 22(b) difference in lobes in the directivity plots for the non-dimensionalized acoustic intensities based on RMS pressure level (scaled by 10 for comparison) and the peak harmonic corresponding to $\mathrm{f}=840 \mathrm{~Hz}$ in Run 1 .

\subsection{Results for selected experimental conditions}

Three cases are selected to compare with results of the acoustic experiments conducted in the ECL low-speed anechoic wind tunnel with NACA0012 airfoil with chord $c=0.1 \mathrm{~m}$ installed at the geometric angle of attack $\alpha=5 \mathrm{deg}$. The 
(a)

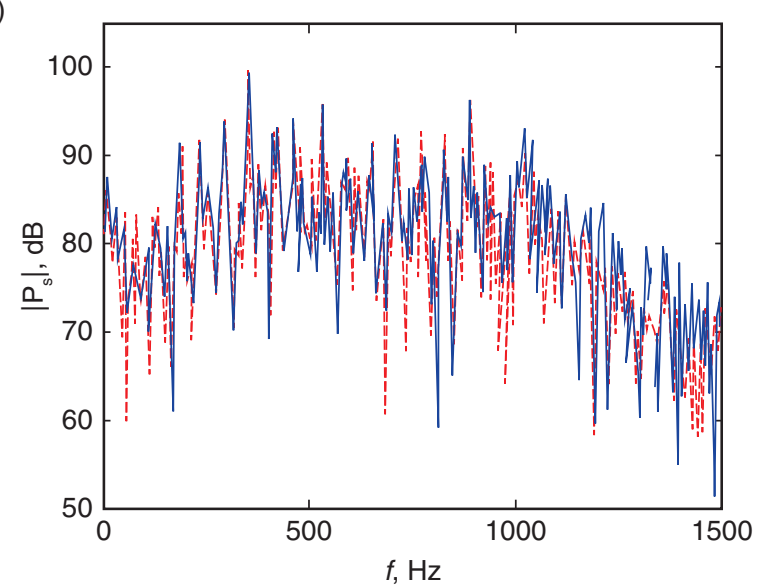

(b)



Figure 22: (a) Pressure spectra at Points 1 (solid blue) and 4 (dashed red); (b) Nondimensionalized directivities of acoustic intensity at $\mathrm{R} / \mathrm{c}=1$ for $\mathrm{f}=840$ $\mathrm{Hz}$ peak harmonic (red) and RMS (scaled by 10, blue) pressure levels.

cases investigated in this numerical study correspond to the mean upstream flow velocities of 20,25 and $30 \mathrm{~m} / \mathrm{sec}$ (denoted, respectively, by Runs 1, 2 and 3 in the following discussion).

The wind tunnel correction of Brooks et $\mathrm{al}^{31}$ for the effective angle of attack in the open air is applied so that $\alpha=2 \mathrm{deg}$ is imposed in the numerical simulations. The physical time step of $0.3625 \times 10^{-6} \mathrm{sec}$ is kept constant in the present analyses. Also similar to the previous validation study, the steady-state flow conditions are reached after marching for 200,000 steps. The pressure signals are then recorded for over 
Table 2. Non-dimensional parameters in numerical computations.

\begin{tabular}{lccc} 
& Run 1 & Run 2 & Run 3 \\
\hline $\mathrm{M}$ & 0.058 & 0.072 & 0.086 \\
$\mathrm{Re}_{1}$ & 38,500 & 48,000 & 57,700 \\
$\mathrm{Re}_{2}$ & & 180,000 & \\
$\Delta \mathrm{t}$ & $7.2 \times 10^{-5}$ & $9.0 \times 10^{-5}$ & $1.1 \times 10^{-4}$
\end{tabular}

720,000 steps thus collecting the data for $0.26 \mathrm{sec}$ with the sampling rate of $62.7 \mathrm{kHz}$. Thus, the same frequency resolution of $\Delta \mathrm{f}=3.83 \mathrm{~Hz}$ is achieved in each case. Table 2 lists the approximated variable non-dimensional flow and numerical parameters employed in each computation. Note that the effect of Reynolds number is examined for Run 2 by considering two sets of values, the first $\left(\operatorname{Re}_{1}\right)$ based on re-scaling from the study of Desquesnes et $\mathrm{al}^{15}$, and the second $\left(\mathrm{Re}_{2}\right)$ more closely representing the actual experimental data.

\subsubsection{Boundary Layer Statistical Moments}

In the numerical analysis, the boundary-layer statistics is collected for the near-wall mean and RMS flow velocities and pressure to gain a better insight in the flow-acoustic interaction process. Results obtained for mean and RMS airfoil surface pressure coefficients shown in Fig. 23 for $\mathrm{Re}_{1}$ values indicate distorted boundary-layer zones on the suction side observed in all the computed cases. This is in apparent contrast with the experimental data for the mean pressure coefficient included in Fig. 23(a). Although the results match very well for the pressure distribution on the pressure side and for the suction side near the leading edge, the distortion-induced hump is not observed in the measurements. On the other hand, as shown in Fig. 24, the results obtained for Run 2 with $\mathrm{Re}_{2}=180,000$ do indicate a significant reduction in the size of the hump. Further analysis is focused solely on Run 2 to observe the effect of Reynolds number on the boundary-layer statistics.

The distribution of the skin friction coefficient on the airfoil surface shown in Fig. 25 gives an important indication of the separated regions visibly localized on the suction side, with their position being rather sensitive to Reynolds number. The results qualitatively resemble those of Desquesnes et $\mathrm{al}^{15}$ but, in contrast, show a very short separated region very close to the trailing edge on the pressure side. Note that the latter was, in fact, linked to the amplification of instabilities and the acoustic feedback as presumed by Desquesnes et $\mathrm{a}^{15}$. The laminar bubble is just slightly more extended for $\operatorname{Re}=180,000$.

Much more pronounced and complex separated zones develop on the suction side in both cases. The case with $\mathrm{Re}=48,000$ reveals a very thin separated region between $x=0.5$ and $x=0.78$ followed by reattachment and immediate separation, with another shorter but much more pronounced laminar bubble extending to $x=0.9$. In the case with $\operatorname{Re}=180,000$, a visibly single separated flow region forms between 
(a)

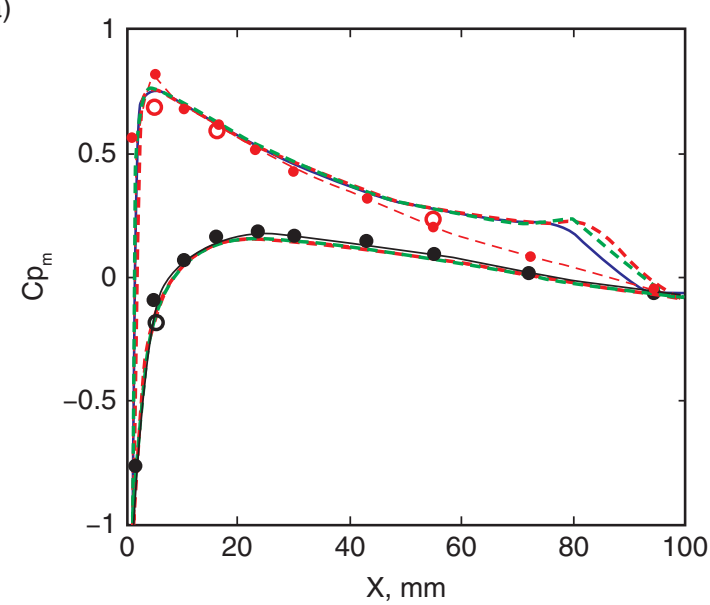

(b)

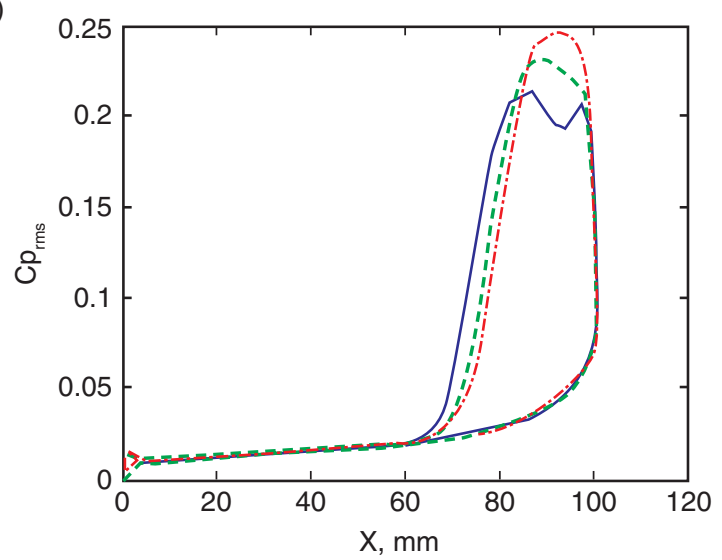

Figure 23: (a) Airfoil surface mean pressure coefficient compared with experimental results (line with markers); (b) Corresponding computed RMS values. $\mathrm{U}=20 \mathrm{~m} / \mathrm{s}$ (solid blue), $25 \mathrm{~m} / \mathrm{s}$ (dashed green), $30 \mathrm{~m} / \mathrm{s}$ (dash-dotted red).

$x=0.5$ and $x=0.78$ starting with a very thin separated layer and followed by a near reattachment point at $x=0.67$ and a shorter and weaker (compared to the previous case) laminar bubble.

The behavior of the boundary-layer statistical moments is further illustrated through comparison of results obtained for the two Reynolds number cases, as shown in Fig. 26 for the suction side. Variations of the mean and RMS of tangential velocity component $V_{t}$, are displayed for seven points on each side between $x=0.7$ and $x=0.95$ (as indicated in the legend). A reversed flow is clearly observed only in the first case accompanied by an extended laminar bubble. Note that the presence of the inflection point indicates possible development of inviscid (Rayleigh) instabilities that tend to 
(a)

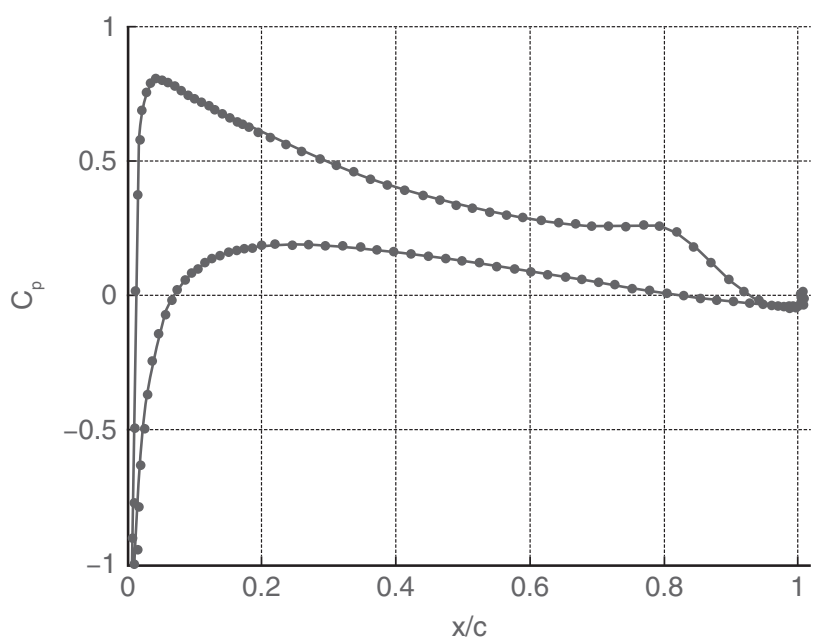

(b)

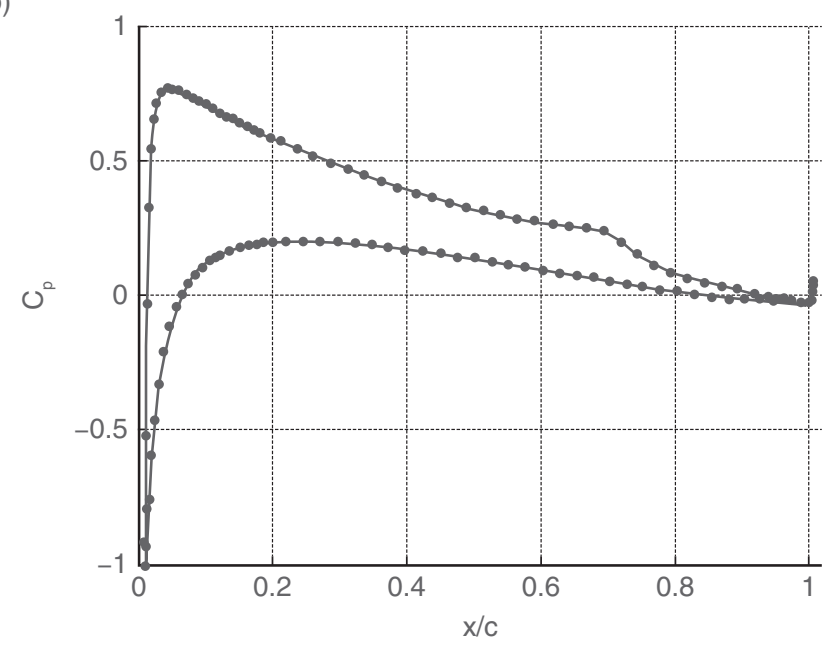

Figure 24: Airfoil surface mean pressure coefficient, $U=25 \mathrm{~m} / \mathrm{s}$. (a) $\mathrm{Re}=48,000$; (b) $\operatorname{Re}=180,000$.

amplify at much higher rate compared to the initially triggered T-S waves. Results for the pressure side (not shown) for each Reynolds number indicate a long but very thin separation region. A critical flow feature corresponding to the multiple-peak RMS velocity profile is noted in Ref. [15] and therein quoted works as characteristic of the developed instability waves but is only observed on the suction side in the current analysis. This is in contrast to the findings of Ref. [15] where results revealed tripleor double-peak RMS velocity behavior on either side, thus leading to the their 
(a)

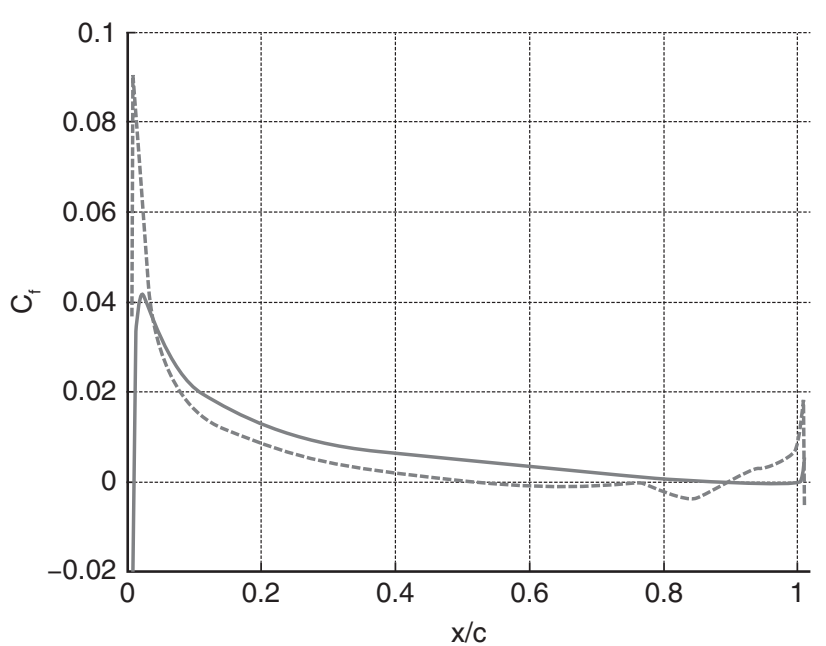

(b)

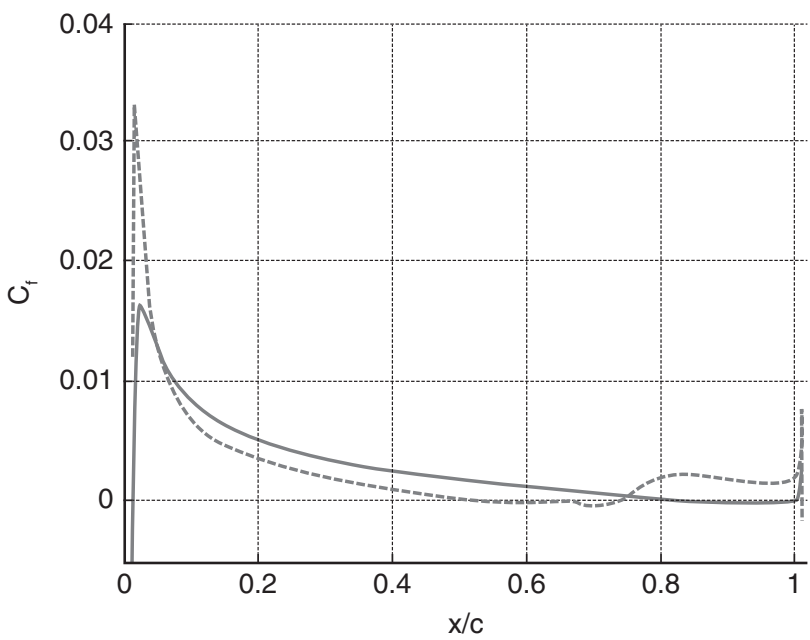

Figure 25: Skin friction coefficient on suction (dashed) and pressure (solid) sides, $U$ $=25 \mathrm{~m} / \mathrm{s}$. (a) $\operatorname{Re}=48,000$, (b) $\operatorname{Re}=180,000$.

conclusion on the interaction of two established feedback loops as an essential mechanism for the tonal trailing-edge noise production ${ }^{15}$.

Finally, to further quantify the growth of the boundary-layer velocity fluctuations, Fig. 27 illustrates the evolution (along the suction and pressure sides) of the RMS maximum for the tangential velocity component $V_{t}$ calculated throughout the boundary layer for each Reynolds number. The growth and saturation on the suction side in Fig. 27(a) is clearly associated with the laminar separation zones formed around $x=0.5$ 
(a)
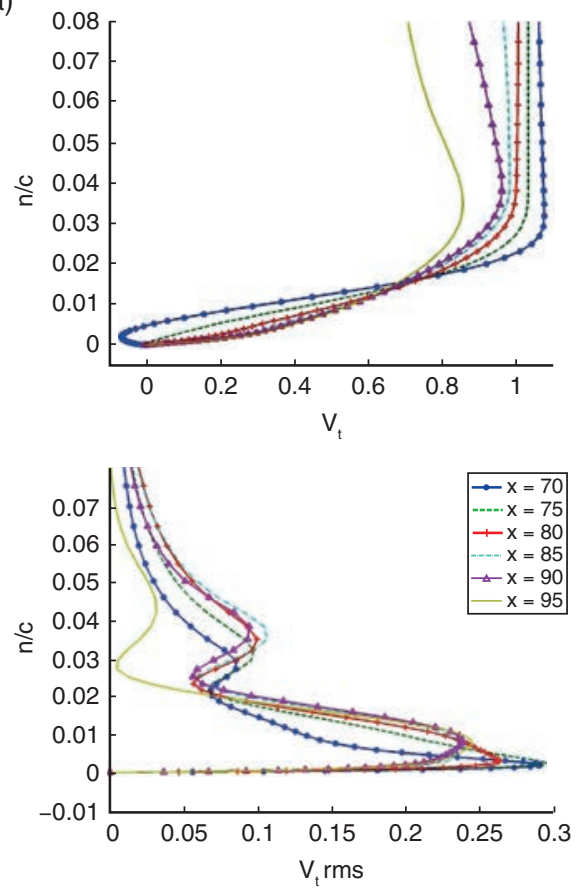

(b)
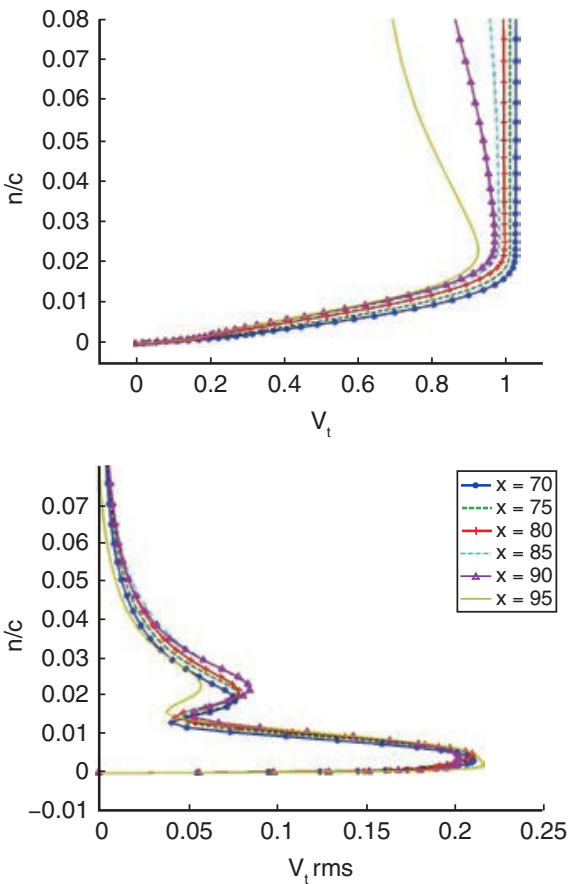

Figure 26: Mean and RMS of tangential velocity on the airfoil suction surface, $\mathrm{U}=25 \mathrm{~m} / \mathrm{s}$. (a) $\mathrm{Re}=48,000$, (b) $\mathrm{Re}=180,000$.

in each case. A more complex double-separation region seen in Fig. 25 contributes to the overall higher levels reached in the case of $\operatorname{Re}=48,000$. Importantly, in each case, the saturation points appear to be associated with the intermediate reattachment locations observed in Fig. 25 immediately upstream of the prominent laminar bubbles. On the pressure side in Fig. 27(b), a rapid growth in RMS maxima is observed very close to the trailing edge where the discussed thin separated layers are formed. Note that similar patterns were also found by Desquesnes et $\mathrm{al}^{15}$. Based on the linear stability analysis, they concluded that the main radiated tonal frequency coincided with that of the most amplified instability mode on the pressure side (rather than the suction side), which contributed to their hypothesis of the main acoustic feedback loop forming there and the secondary one appearing on the suction side. However, while the assumption of dual interacting loops may appear possible under certain scenarios, our experimental study involving the pressure-side tripped boundary layer shows that the suction side alone can sustain the acoustic feedback with pronounced sound radiation (Fig. 8). Furthermore, the current results of the spectral analysis shown below suggest that the unsteady response is overall higher on the suction side. On the other hand, one may note that the presented RMS and spectral data does not differentiate between the convected instability and acoustic wave contributions to the overall response signature. 
(a)

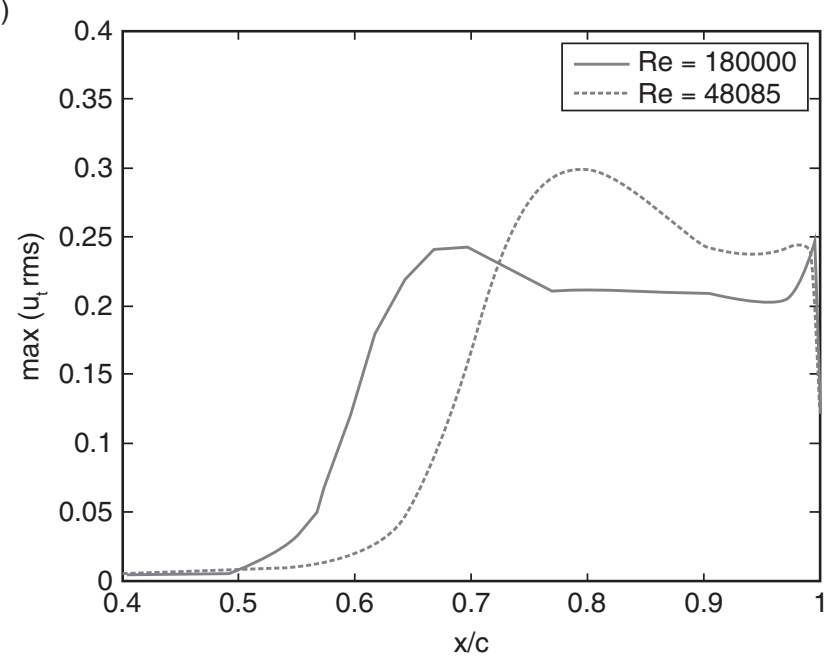

(b)

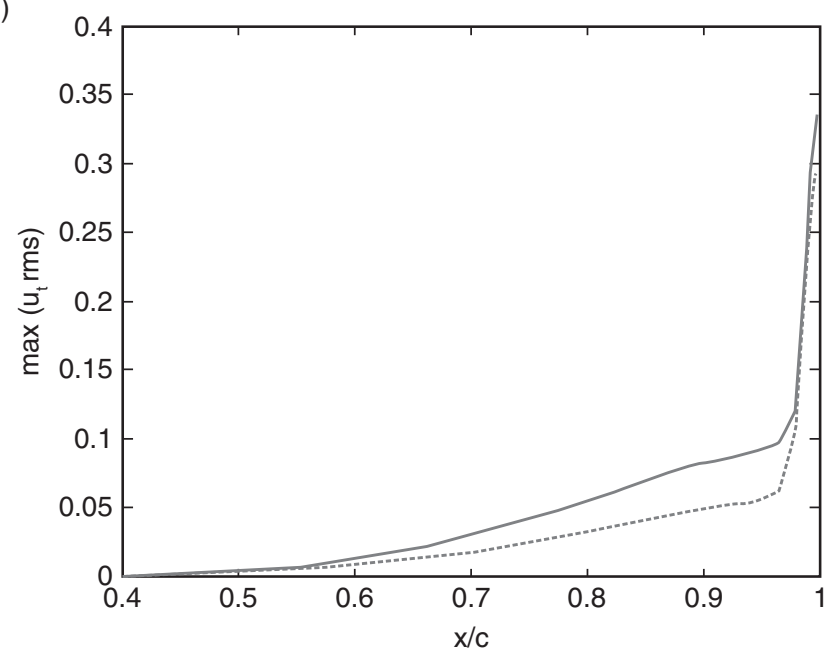

Figure 27: Evolution of RMS maxima for tangential velocity on the airfoil (a) suction and (b) pressure sides, $U=25 \mathrm{~m} / \mathrm{s}$.

A procedure is currently developed to provide an adequate source identification for the obtained velocity and pressure amplitudes.

\subsubsection{Spectral Analysis}

Fig. 28 illustrates the instantaneous near-field vorticity and pressure contours obtained from 2D numerical simulation conducted for Run 2 for $\mathrm{Re}=48,000$. FFT analysis of the sampled pressure signal has been conducted at the selected in the experiment remote-microphone probe locations on the airfoil surface shown in Fig. 9. The 


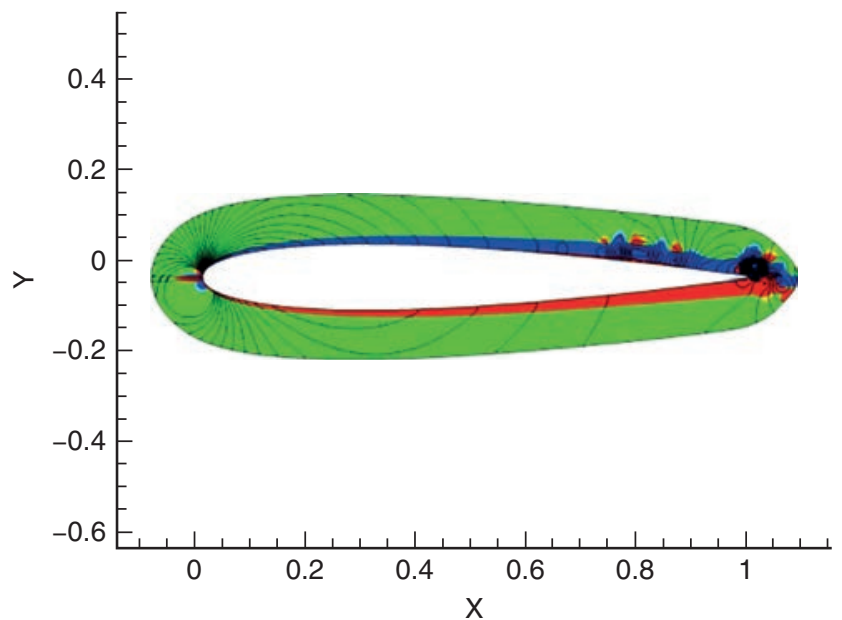

Figure 28: (a) Vorticity (colored) and pressure contours, $U=25 \mathrm{~m} / \mathrm{sec}, \mathrm{Re}=48,000$.

comparison is presented both for $\operatorname{Re}=48,000$ and $\operatorname{Re}=180,000$ cases against experimental results. In addition, the near-field numerical data correction per Oberai et $\mathrm{al}^{32}$ is applied to compare with the acoustic measurement at the microphone location in the far field. In particular, for the acoustic spectra, such correction indicates 8-11 dB difference in the range of 500-1000 Hz between 2D and 3D predictions with account for the near-field position of Point 1 (where the numerical data is collected) relative to the airfoil trailing edge and the microphone location. For an estimate, a constant shift of $-10 \mathrm{~dB}$ is applied across the spectrum to $2 \mathrm{D}$ results to reveal the measured peak value close to the corresponding numerical values.

Overall, the comparison of the obtained results at the probe locations in Fig. 29 shows significant differences in the spectral distributions. In particular, the broadband levels predicted from 2D numerical simulations are much higher and nearly absorb spectral peaks which, in contrast, appear to be distinctly superimposed on the broadband humps in the measured data (with the pattern repeated for higher harmonics). This discrepancy points to the need to employ fully 3D numerical simulations to resolve the occurring spanwise energy redistribution in the transitional boundary layer, with corresponding results to be reported in the future work.

The obtained 2D numerical results appear similar in shape and amplitude for the two Reynolds numbers but the differences are notable. At point $\mathrm{B}$, results for $\mathrm{Re}=180,000$ show higher spectral levels in agreement with the more rapidly developed, higher maximum RMS of $V_{t}$ in Fig. 27(a). The opposite occurs at all other locations (especially at point $\mathrm{A}$ at the trailing edge) but in the far field both cases appear nearly on par with each other. Note that the spectra at points A and D on the suction side were compared with corresponding points on the pressure side (with the same locations along the chord). The levels appeared much higher near the trailing edge on the suction side thus again stressing the suspected dominant contribution from the acoustic feedback formed 

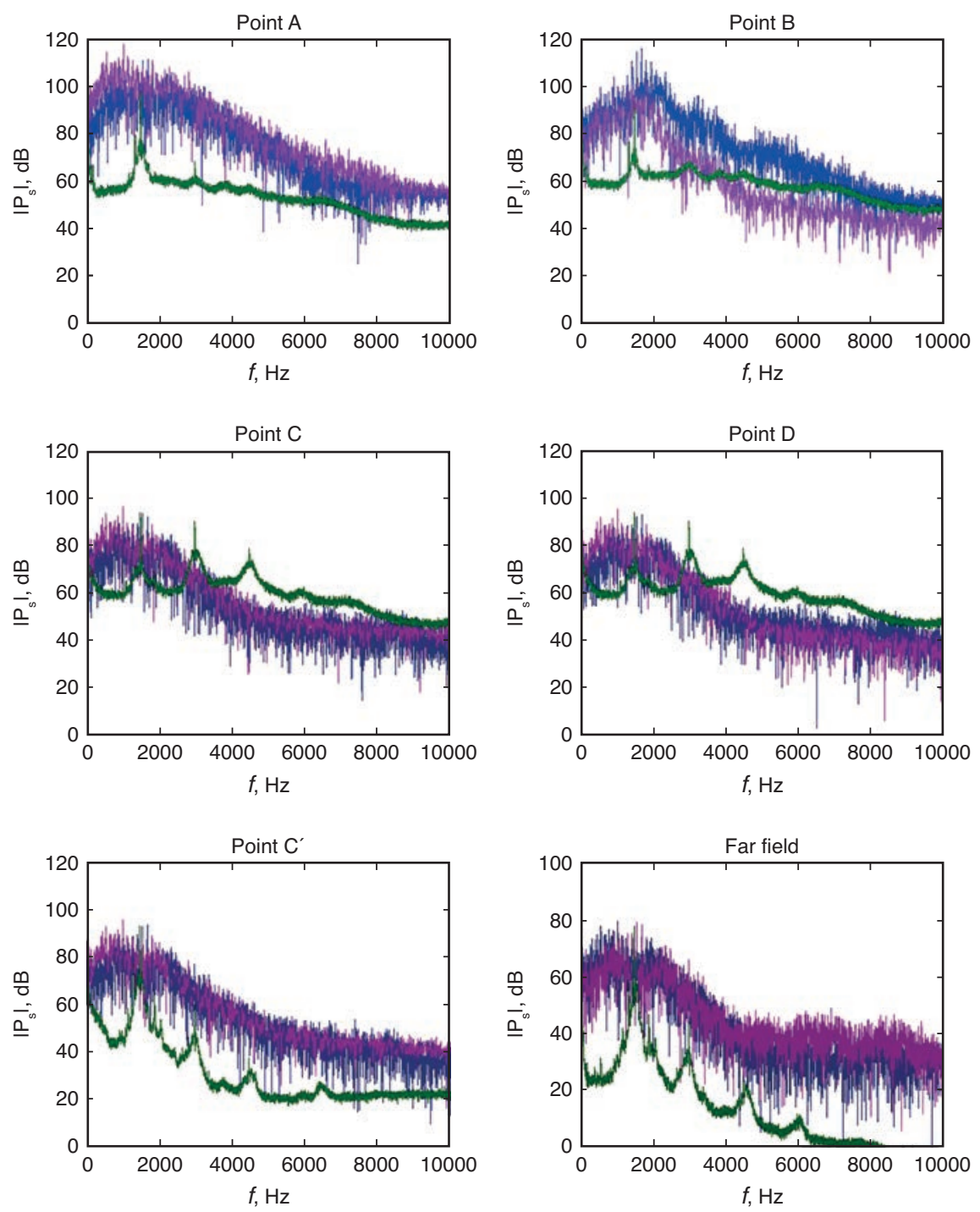

Figure 29: Comparison of predicted (magenta for $\operatorname{Re}=48,000$, blue for $\operatorname{Re}=180,000$ ) vs. measured (green) airfoil surface and far-field pressure spectra, $\mathrm{U}=25 \mathrm{~m} / \mathrm{s}$.

on that side of the airfoil. It may be also concluded that the elevated spectrum at point A could reflect high level of aerodynamic fluctuations in the distorted region since the spectral levels at the corresponding upstream points are nearly identical on the pressure and suctions sides and thus appear to be determined by acoustic fluctuations. 


\subsubsection{Acoustic Feedback Tone Selection}

The clusters of equally spaced tones observed in Fig. 29 are believed to be associated with the self-sustained acoustic feedback loop mechanism involving downstreamconvected boundary-layer instability modes and the upstream-propagating trailing-edge acoustic waves. To validate this assumption, the tonal frequencies are matched against the feedback-loop relationship proposed by Arbey \& Bataille ${ }^{5}$,

$$
f\left(\frac{L}{U_{c}}+\frac{L}{c_{o}-U_{c}}\right)=\left(n+\frac{1}{2}\right)
$$

where the tonal frequency $f$ is related to the modal number $n$, distance $L$ between the trailing edge and the instability wave inception point (typically, the point of maximum velocity on the surface), the wave convection speed $U_{c}$, and the speed of sound $c_{0}$. The results of such frequency selection for the most prominent tones in the spectra are shown in Table 3 for the cases with $U=25 \mathrm{~m} / \mathrm{sec}$ and $30 \mathrm{~m} / \mathrm{sec}$ (with $\operatorname{Re}_{1}$ values in Table 2 ), and the estimates employed the values of $L=0.095 \mathrm{~m}$ (based on the point with minimum surface pressure), $U_{c} \approx 0.47 \mathrm{U}$, and $c_{0}=340 \mathrm{~m} / \mathrm{sec}$. The numerically predicted tones show an excellent comparison with those obtained from the equation above. Furthermore, the computed frequency spacing between the tones perfectly matches with the theoretical formula for the feedback loop $\Delta \mathrm{f}=K U^{m} / L$, for $K=0.86$ and $m=0.8$. The latter values are known to correspond to the mechanism of scattered instability waves which combined radiation contributes to a characteristic dipole-type pattern of the trailing-edge noise (observed, e.g., in Fig. 22(b)). It is important to note that while the employed 2D numerical analysis appears to overpredict the broadband spectra compared to the experimental data, it is believed to accurately identify dominant tonal frequencies associated with the acoustic feedback loop (a similar assumption was made, e.g., in Ref. [33] for the feedback loop in cavity noise studies).

Table 3. Computed vs. predicted (Eq. (1)) tonal frequencies for $25 \mathrm{~m} / \mathrm{sec}$ (left) and $30 \mathrm{~m} / \mathrm{sec}$ (right)

\begin{tabular}{lrrr}
$\mathbf{2 5} \mathbf{~ m} / \mathbf{s e c}$ & $\mathbf{n = 7}$ & $\mathbf{n = 8}$ & $\mathbf{n = 9}$ \\
\hline$f, \mathrm{~Hz}$ computed & 892 & 1011 & 1130 \\
$f, \mathrm{~Hz}$ & 892 & 1011 & 1130 \\
predicted, Eq.1 & & &
\end{tabular}

\begin{tabular}{lrrr}
$\mathbf{3 0} \mathbf{~ m} / \mathbf{s e c}$ & $\mathbf{n}=\mathbf{6}$ & $\mathbf{n}=\mathbf{7}$ & $\mathbf{n}=\mathbf{8}$ \\
\hline$f, \mathrm{~Hz}$ computed & 941 & 1080 & 1221 \\
$f, \mathrm{~Hz}$ & 934 & 1078 & 1221 \\
predicted, Eq.1 & & &
\end{tabular}




\section{CONCLUSIONS}

This work reported on results of recent experimental and numerical studies of flowacoustic interactions in transitional airfoils and their impact on airfoil surface unsteady pressure and acoustic signature. Tests were conducted with NACA0012 rectangular airfoil sections of $8 \mathrm{~cm}$ and $10 \mathrm{~cm}$ chords and of $30 \mathrm{~cm}$ span (aspect ratio of nearly 3), both in clean flow and with controlled inflow disturbance, with boundary-layer tripping (on one or both sides) and without tripping, at different angles of attack, and for a range of flow speeds corresponding to $M=0.03 \ldots 0.15$ $\left(R e_{c}=0.5 \times 10^{5} \ldots 3 \times 10^{5}\right)$.

With the clean upstream flow conditions, the experiments revealed a ladder-type structure of acoustic tones with dual velocity dependence. They corresponded to the rungs with frequency $f \sim U^{0.8 \ldots 0.85}$ related to the amplified instability-wave trailingedge scattering, and the effectively produced vorticity shedding corresponding to the dominant frequencies of each rung scaled with $f_{s} \sim U^{1.5}$. Airfoil surface tripping revealed different levels of contributions to the resulting pressure spectra induced by pressure- or suction-side boundary-layer instabilities depending on flow velocity. Numerical results deviated from measurements in pressure spectra but revealed a pattern of multiple tones matching well with the formula for the acoustic feedback loop mechanism.

The effect of weak upstream turbulence on the radiated sound was studied experimentally. The main effect was to suppress all tones associated with acoustic feedback and the 0.85-power ladder-type structure. But the main trace of the hump corresponding to the primary vorticity-shedding mechanism of the trailing-edge acoustic radiation was preserved and still followed the 1.5-power law.

\section{ACKNOWLEDGEMENTS}

The authors would like to acknowledge support for this work through Air Force Office of Scientific Research (Award Number FA9550-12-1-0126, Program Manager Dr. D. Smith), and Florida Center for Advanced Aero Propulsion. The authors are also grateful to M. Jacob (ECL) for help in postprocessing results, to E. Jondeau (ECL) for his participation in the experiments, and to Centrale Innovation for partial support of the project.

\section{REFERENCES}

[1] Golubev, V.V., Dreyer, B.D., Hollenshade, T.M., Visbal, M.R., (2009), "HighAccuracy Viscous Analysis of Unsteady Flexible Airfoil Response to Impinging Gust," AIAA Paper 2009-3271, $15^{\text {th }}$ Joint AIAA/CEAS Aerocoustics Conference, Miami, May 2009.

[2] Golubev, V.V., Nguyen, L., Visbal, M.R., (2010), "High-Fidelity Viscous Simulations of Airfoil Noise Radiation in Nonuniform Unsteady Flow," AIAA 2010-3761, 16th AIAA/CEAS Aeroacoustics Conference, Stockholm, Sweden, June 2010.

[3] Paterson, R., Vogt, P., Fink, M., Munch, C., (1973), "Vortex Noise of Isolated Airfoils," J. Aircraft, Vol. 10, pp. 296-302. 
[4] Tam, C.K.W., (1974), "Descrete Tones of Isolated Airfoils," Journal of Acoustic Society of America, Vol. 55, pp. 1173-1177.

[5] Arbey, H., Bataille, J., (1983), "Noise Generated by Airfoil Profiles Placed in a Uniform Laminar Flow,” J. Fluid Mechanics, Vol. 134, pp. 33-47

[6] Longhouse, R.E., (1977), "Vortex Shedding Noise of Low Tip Speed Axial Flow Fans," Journal of Sound and Vibration, Vol. 53, pp. 25-46.

[7] Atassi, H.M., (1984), "Feedback in Separated Flows over Symmetric Airfoil," NASA-TM-83758.

[8] Lowson, M.V., Fiddes, S.P., Nash, E.C., (1994), "Laminar Boundary Layer Aeroacoustic Instabilities," AIAA Paper 94-0358.

[9] Nash, E.C., Lowson, M.V., McAlpine, A., (1999), "Boundary Layer Instability Noise on Airfoils," J. Fluid Mechanics, Vol. 382, pp.27-61.

[10] Obremsky, H.J., Morkovin, M.V., Landahl, M., Wazzan, A.R., Okamura, T.T., Smith, A.M.O., (1969), "A Portfolio of Stability Characteristics of Incompressible Boundary Layers,” AGARDograph 134.

[11] McAlpine, A., Nash, E.C., Lowson, M.V., (1999), "On the Generation of Descrete Frequency Tones by the Airflow Around an Airfoil," Journal of Sound and Vibration, Vol. 222, pp. 753-779.

[12] Koch, W., (1985), "Local Instability Characteristics and Frequency Determination of Self-Excited Wake Flows," Journal of Sound and Vibration, Vol. 99, pp. 53-83.

[13] Jones, L.E., Sandberg, R.D., Sandham, N.D., (2008), "Direct Numerical Simulations of Forced and Unforced Separation Bubble on an Airfoil at Incidence," Journal of Fluid Mechanics, Vol. 602, pp. 175-207.

[14] Kingan, M.J., Pearse, J.B., (2009), "Laminar Boundary Layer Instability Noise Produced by an Airfoil," Journal of Sound and Vibration, Vol. 322, pp. 808-828.

[15] Desquesnes, M., Terracol, M., Sagaut, P., (2007), "Numerical Investigation of the Tone Noise Mechanism over Laminar Airfoils," J. Fluid Mechanics, Vol. 591, pp. 155-182.

[16] Brooks, T.F., Pope, D.S., Marcolini, M.A., (1989), “Airfoil Self-Noise and Prediction," NASA RP-1218, pp.1-137.

[17] Visbal, M.R., Gaitonde, D.V., (2002), "On the Use of High-Order FiniteDifference Schemes on Curvilinear and Deforming Meshes," Journal of Computational Physics, Vol. 181, pp.155-185.

[18] Visbal, M.R., Rizzetta, D.P., (2002), "Large-Eddy Simulation on Curvilinear Grids Using Compact Differencing and Filtering Schemes," Journal of Fluids Engineering, Vol. 124, pp. 836-847.

[19] Visbal, M.R., Morgan, P.E., Rizzetta, D.P., (2003), “An Implicit LES Approach Based on High-Order Compact Differencing and Filtering Schemes," AIAA Paper 2003-4098.

[20] Visbal, M.R., Gordnier, R.E., Galbraith, M.C., (2009), "High-Fidelity Simulations of Moving and Flexible Airfoil at Low Reynolds Numbers," Experiments in Fluids, Vol. 46, pp. 903-922. 
[21] Golubev, V.V., Dreyer, B.D., Hollenshade, T.M., Visbal, M.R., (2009), "HighAccuracy Viscous Simulation of Gust Interaction with Stationary and Pitching Wing Sections," AIAA Paper 2009-0011, 47 $7^{\text {th }}$ AIAA Aerospace Sciences Meeting and Exhibit, Orlando, January 2009.

[22] Golubev, V.V., Dreyer, B.D., Hollenshade, T.M. Visbal, M.R., (2009), "HighAccuracy Viscous Simulation of Gust-Airfoil Nonlinear Aeroelastic Interaction," AIAA Paper 2009-4200, 39 ${ }^{\text {th }}$ AIAA Fluid Dynamics Conference, San Antonio, June 2009.

[23] Golubev, V.V., Hollenshade, T.M., Nguyen, L., Visbal, M.R., (2010), "Parametric Viscous Analysis of Gust Interaction with SD7003 Airfoil," AIAA Paper 2010928, 48 ${ }^{\text {th }}$ AIAA Aerospace Sciences Meeting and Exhibit, Orlando, January 2010.

[24] Golubev, V.V., Nguyen, L., Visbal, M.R., (2010), "High-Accuracy Low-Re Simulations of Airfoil-Gust and Airfoil-Vortex Interactions," AIAA Paper 2010-4868, 40 ${ }^{\text {th }}$ AIAA Fluid Dynamics Conference, Chicago, June 2010.

[25] Golubev, V.V., Nguyen, L., Visbal, M.R., (2011), "High-Fidelity Simulations of Transitional Airfoil Interaction with Upstream Vortical Structure," AIAA Paper 2011-394, 49 $9^{\text {th }}$ AIAA Aerospace Sciences Meeting and Exhibit, Orlando, January 2011.

[26] Wagner, C., Huttl, T., Sagaut, P., (2007), "Large-Eddy Simulation for Acoustics," Cambridge University Press.

[27] Golubev, V.V., Brodnick, J., Nguyen, L., Visbal, M.R., (2011), "New Approach to Modeling Airfoil Interaction with Upstream Turbulence," AIAA Paper 20113899, 41 ${ }^{\text {th }}$ AIAA Fluid Dynamics Conference, Honolulu, June 2011.

[28] Smirnov, R., Shi, S., Celik, I., (2001), "Random Flow Generation Technique for Large Eddy Simulations and Particle-Dynamics Modeling," Journal of Fluids Engineering, Vol. 123, pp.359-371.

[29] Huang, S.H., Li, Q.S., Wu, J.R., (2010), “A General Inflow Turbulence Generator for Large Eddy Simulation," Journal of Wind Engineering and Industrial Aerodynamics, Vol. 98, pp. 600-617.

[30] Moreau, S., Henner, M., Iaccarino, G., Wang, M., Roger, M., (2003), “Analysis of Flow Conditions in Freejet Experiments for Studying Airfoil Self-Noise," AIAA Journal, Vol.41, pp. 1895-1905.

[31] Brooks, T.F., Marcolini, M.A., Pope, D.S., (1984), “Airfoil Trailing Edge Flow Measurements and Comparison with Theory Incorporating Open Wind Tunnel Corrections," AIAA Paper 84-2266.

[32] Oberai, A.A., Roknaldin, F., Hughes, T.J.R., (2002), "Computation of TrailingEdge Noise Due to Turbulent Flow Over an Airfoil," AIAA Journal, Vol. 40, pp. 2206-2216.

[33] Rowley, C.W., Colonius, T., Basu, A.J., (2002), "Self-Sustained Oscillations in Two-Dimensional Compressible Flow Over Rectangular Cavities," Journal of Fluid Mechanics, Vol. 455, pp. 315-346. 